\title{
Wall turbulence response to surface cooling and formation of strongly stable stratified boundary layers
}

Cite as: Phys. Fluids 31, 085114 (2019); https://doi.org/10.1063/1.5109797

Submitted: 13 May 2019 • Accepted: 30 July 2019 • Published Online: 20 August 2019

Amir Atoufi, K. Andrea Scott and Michael L. Waite

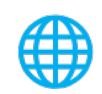

\section{ARTICLES YOU MAY BE INTERESTED IN}

Direct numerical simulation and statistical analysis of stress-driven turbulent Couette flow with a free-slip boundary

Physics of Fluids 31, 085113 (2019); https://doi.org/10.1063/1.5099650

Effects of bulk viscosity on compressible homogeneous turbulence

Physics of Fluids 31, 085115 (2019); https://doi.org/10.1063/1.5111062

Coherent structures in tornado-like vortices

Physics of Fluids 31, 085118 (2019); https://doi.org/10.1063/1.5111530

\section{Physics of Fluids}




\title{
Wall turbulence response to surface cooling and formation of strongly stable stratified boundary layers
}

\author{
Cite as: Phys. Fluids 31, 085114 (2019); doi: 10.1063/1.5109797 \\ Submitted: 13 May 2019 • Accepted: 30 July 2019• \\ Published Online: 20 August 2019
}

Amir Atoufi, $^{1,2, a)}$ (D) K. Andrea Scott, ${ }^{7}$ and Michael L. Waite ${ }^{2}$

\author{
AFFILIATIONS \\ ${ }^{1}$ Department of Systems Design Engineering, University of Waterloo, Waterloo, Ontario N2L 3G1, Canada \\ ${ }^{2}$ Department of Applied Mathematics, University of Waterloo, Waterloo, Ontario N2L 3G1, Canada \\ a)aatoufi@uwaterloo.ca
}

\begin{abstract}
This paper investigates the processes by which stable boundary layers are formed through strong surface cooling imposed on neutrally stratified wall-bounded turbulence using high-resolution direct numerical simulation at a moderate Reynolds number. The adjustment of the flow to the imposed strong surface cooling is investigated. We further focus on a strongly stable case where turbulence partially collapses. We show that, due to a significant reduction in turbulence production, turbulence becomes patchy, with a band of turbulence coexisting with quiet regions. The nature of the quiet regions, which are often characterized as laminar, is investigated and shown to be consistent with viscously coupled stratified turbulence. The one-dimensional longitudinal streamwise velocity spectrum exhibits $k_{x}^{-5}$ and $k_{x}^{-3}$ behavior in the buffer and logarithmic layers, respectively, adjacent to an active region of three-dimensional turbulence with a $k_{x}^{-5 / 3}$ spectrum. Scenarios for turbulence recovery from such a patchy state are also discussed. We show that the presence of outer layer turbulence above $z^{+} \approx 300$ is a key requirement for recovery. For higher values of stratification, it is shown that inner layer turbulence is damped entirely and outer layer turbulence is damped subsequently.
\end{abstract}

\section{Published under license by AIP Publishing. https://doi.org/10.1063/1.5109797}

\section{INTRODUCTION}

Turbulence plays a central role in the dynamics of the atmospheric boundary layer (ABL), e.g., in the mixing of heat and water vapor, dispersion of air pollutants, and cloud formation. However, stable stratification, in which heavier air lies under lighter air, significantly affects boundary layer turbulence. ${ }^{1-4}$ Strongly stable stratification may lead to coexistence of turbulence and internal waves, $3,5,6$ horizontal intermittency, ${ }^{7}$ or oblique laminar-turbulent patterns. ${ }^{8}$ These features of the strongly stable regime demand extra consideration and add additional challenges for numerical simulations (e.g., microfronts ${ }^{3}$ and potential collapse of turbulence ${ }^{4}$ ). Because of the complexity of the stably stratified regime, it presents a challenge for parameterization of boundary layer mixing in weather forecasting models.

The stably stratified boundary layer (SBL) can form by radiative cooling of the surface (e.g., nocturnal ABL) or by advection of warm air over cold surfaces (e.g., ABL over sea ice). From an environmental perspective, the SBL can lead to a decrease in the dispersion of air pollutants or an accumulation of aerosols (e.g., black carbon ${ }^{9}$ ) in near-surface regions. ${ }^{10,11}$ The SBL can also adversely impact the local ecology by rapid depletion of nutrients due to the decrease in surface layer mixing. ${ }^{12,13}$ From the perspective of wind energy, stable stratification can cause a significant reduction in the total power output of large wind farms. ${ }^{14}$

In the SBL, the dynamics of turbulence and the energy cascade are controlled by the simultaneous effects of stable stratification and the solid boundary. For stable stratification, energy is transferred from large anisotropic eddies to smaller and more isotropic eddies. In particular, for scales above the Ozmidov scale, anisotropy is characterized by thin pancake vortices, layers of strong shear, and gravity waves; below the Ozmidov scale, there is a transition to more isotropic three-dimensional turbulence. ${ }^{15-17}$ The presence of the wall, on the other hand, creates another source of anisotropy by 
which the size of the energy-containing eddies increases when moving away from the wall. This causes an inverse spatial cascade in the near-wall region where energy and momentum are transferred from smaller eddies to larger eddies aloft. ${ }^{18,19}$

The SBL has been the subject of numerous studies by numerical simulations and atmospheric measurements and observations. However, the interplay of the solid boundary and stratified turbulence, particularly for strong stratification, has been less studied. Some of the outcomes of these studies of stably stratified wallbounded turbulence (mostly from numerical simulations of closed or open channel flow) are briefly reviewed here.

SBL flows can be classified into "buoyancy affected," "buoyancy controlled," or "buoyancy dominated" based on Richardson and Reynolds numbers. ${ }^{20}$ SBL turbulence is sustained for weak stratification or a high Reynolds number (buoyancy affected flows). 5,7,20-23 Nevertheless, stratification substantially affects turbulence in the outer layer, while the near-wall region and the logarithmic layer remain almost unaffected. ${ }^{21,23}$ For moderate stratification (buoyancy controlled flows), symmetry around the center of the closed channel is broken. ${ }^{20}$ However, symmetry is eventually restored through a transition process by eddies crossing over the channel center. ${ }^{20}$ Open channel flows at moderate stratification have lower turbulent mass flux, lower density gradient, and higher values for the ratio of potential energy to vertical turbulent kinetic energy ${ }^{21}$ compared to the weak stratification regime.

For strong stratification or a low Reynolds number (buoyancy dominated flows), the turbulence production mechanism is significantly suppressed by stratification, leading to decoupling of the inner and outer layers ${ }^{20}$ or formation of large laminar patches in the near-wall region ${ }^{7,8,23,24}$ (depending on the stratification level) where strongly stable stratification damps the intensity of near-wall streaks $^{5,23}$ generated from global modes. ${ }^{23}$ The damping of these modes eventually leads to vanishing of turbulent momentum and buoyancy fluxes in the core of the channel due to the disappearance of large-scale streaks in the near-wall region. ${ }^{23}$ The dominance of internal gravity waves in the central region has also been observed for buoyancy dominated flows in closed channel simulations. ${ }^{5,6,23}$

Although flow characteristics are fundamentally different for each of these three stratification regimes, they have some common basic features. For example, increasing stratification leads to an increase in the mean streamwise velocity, a decrease in the skin friction coefficient, a decrease in vertical velocity fluctuations, and a decrease in tangential Reynolds stress. ${ }^{5,21-25}$

In the buoyancy dominated SBL, the ratio of the MoninObhukov (MO) length to the channel height is often used to indicate whether turbulence will survive or collapse. ${ }^{7,22}$ The MO scale $L_{M O}$ is defined as

$$
\frac{L_{M O}}{h}=-\frac{u_{\tau}^{3} /(\kappa h)}{\left.\left(g / \theta_{0}\right) \overline{\theta w}\right|_{s}},
$$

where $u_{\tau}$ is the friction velocity based on the wall shear stress, $\kappa \approx 0.41$ is the von Kármán constant, $h$ is a reference length scale (channel height for open channel flow configuration and channel half height for closed channel), and $\left.\overline{\theta w}\right|_{s}$ is the vertical temperature flux at the bottom surface, where the overbar denotes a horizontal average. Essentially, $L_{M O}$ is the height at which the temperature flux at the bottom surface is of the same order as the turbulent energy production in the log-law region, where stratification effects are assumed to be small.

Nieuwstadt ${ }^{22}$ performed pioneering direct numerical simulations (DNSs) of a turbulent open channel flow with a uniformly cooled bottom surface and fixed-temperature upper surface and introduced $h / L_{M O}$ as a stability parameter. He reported that turbulence survives only for $h / L_{M O}<1.25$, for which most locations in the channel satisfy $R i_{g}^{h}<0.25$, where the gradient Richardson number $R i_{g}^{h}=N^{2} / S^{2}$ is the squared ratio between the buoyancy frequency $N$ and mean shear $S$ averaged over horizontal planes. He showed that turbulence decays for larger values of $h / L_{M O}$. Similar criteria have been found for turbulence collapse at higher Reynolds numbers and larger computational domain sizes.

Flores and Riley ${ }^{7}$ performed DNS of a case similar to Nieuw$\operatorname{stadt}^{22}$ at higher Reynolds and Richardson numbers as an idealized model for the nocturnal atmospheric surface layer. They found that surface cooling causes the turbulence statistics near the ground to adjust on a time scale on the order of $L_{M O} / u_{\tau}$. They found that turbulence is sustained for relatively weak cooling, but when the Monin-Obukhov Reynolds number $R e_{L_{M O}}=L_{M O} u_{\tau} / v$ falls below around 100, turbulence collapses. Here, $v$ is the kinematic viscosity. This criterion implies that the logarithmic layer is not large enough to accommodate a buffer region beneath when $R e_{L_{M O}} \lesssim 100$ since the height of the buffer region is about $100 v / u_{\tau}$. A similar study ${ }^{27}$ related turbulence collapse as a local process, regardless of the outer region dynamics. To reach that conclusion, they simulated a capped Ekman layer, which has a different outer flow and found similar criteria.

Donda et al. ${ }^{28,29}$ also performed DNS of a open channel flow with a setup similar to the work of Nieuwstadt ${ }^{22}$ and showed that recovery of turbulence would occur if perturbations of finite amplitude are imposed on the laminarized state after sufficient time. They argued that in the SBL, the available momentum in the bulk of the flow limits the downward heat flux to a maximum, which they called the maximum sustainable heat flux (MSHF). This limit is due to the fact that the time scale of the boundary layer diffusion is much smaller than the time scale for flow acceleration ${ }^{30}$ and therefore the available momentum for downward heat transport is limited in the case of strong surface cooling. ${ }^{30}$ For surface cooling rates larger than MSHF, efficient vertical heat transport hinders and eventually causes turbulence to be suppressed fully by the intensive density stratification.

The focus of these previous studies has been the stationary SBL when turbulence is strong enough to survive surface cooling $5,20,21,23$ and/or the determination of conditions for turbulence collapse. $^{7,22,26-29}$ Another question that has been less discussed in the literature is how a neutrally stratified boundary layer transforms to a SBL through surface cooling. This question is of particular relevance to the formation of a nocturnal ABL. Answering this question can improve our understanding of (1) the process under which turbulence collapses for strongly stable stratification and (2) the mechanism by which turbulence may or may not recover from such collapse. Addressing these questions is the central focus of this paper.

In this paper, we aim to study how a neutral boundary layer evolves into a SBL in the presence of surface cooling. In particular, we investigate how wall turbulence responds to strong surface cooling, how patchiness appears through adjustment of wall turbulence 
to strong stratification, and how turbulence may recover from such patchy states. We also consider the properties of the patchy state, when it is present, and how wall-bounded turbulence is laminarized for very strong stratification.

This paper starts with a general statement of the problem in Sec. II where the mathematical formulation, governing equations, dimensionless parameters, and boundary conditions are introduced. The computational approach, simulation parameters, and properties of the database generated in this investigation are discussed in Sec. III. In Sec. IV, simulation results are presented. First, we describe the feedback process over which the flow responds to strong surface cooling. Then, possible feedback mechanisms that may be responsible for flow adjustment are considered. The paper ends with concluding remarks in Sec. V.

\section{PROBLEM FORMULATION}

In this section, we describe the case study, present the governing equations, boundary conditions, and nondimensionalized parameters, and outline the different layers of the stratified boundary layer.

\section{A. Test case description}

The motivation for the present work is to study turbulence in the surface layer of a stably stratified dry ABL. To do so, an open channel flow configuration is considered, with an imposed cooling flux at the lower boundary and an adiabatic upper boundary. The geometry of the open channel is shown in Fig. 1. A uniform pressure gradient drives flow in the $x$ direction, periodic boundary conditions are applied in both horizontal directions, while no-slip and no-stress surfaces are applied to the bottom and top boundaries, respectively. The $y$ axis is the cross-stream direction, and the $z$ axis is normal to the wall. The velocities in the $x, y$, and $z$ directions are denoted by $u$, $v$, and $w$, and we let $\left(x_{1}, x_{2}, x_{3}\right)=(x, y, z)$ and $\left(u_{1}, u_{2}, u_{3}\right)=(u, v, w)$. The domain size in the $x$ and $y$ directions is $L_{x}$ and $L_{y}$, respectively, and the channel depth is $h$. The temperature field is denoted by $\theta$ and is defined as the fluctuation from background $\theta_{0}$. For simplicity, the free surface is assumed to be undeformed, an approximation applicable for a low Froude number ${ }^{31-34}$ which is a common approach in studies of the idealized ABL. ${ }^{7,2}$

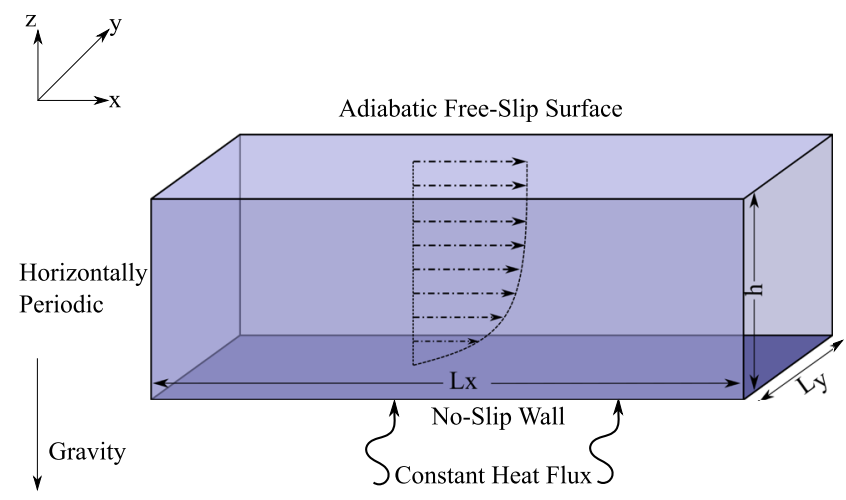

FIG. 1. Stratified open channel configuration.

\section{B. Governing equations and dimensionless parameters}

The governing equations for this work are the Navier-Stokes equations under Oberbeck-Bousinesq approximation (OBA). The OBA equations can be normalized using the channel height $h$, a reference velocity $u_{0}$, and the value of the imposed bottom-surface temperature gradient $F$. With these choices, the dimensionless OBA equations can be written as ${ }^{21,24}$

$$
\begin{aligned}
& \frac{\partial u_{i}}{\partial x_{i}}=0, \\
& \frac{\partial u_{i}}{\partial t}+u_{j} \frac{\partial u_{i}}{\partial x_{j}}=-\frac{\partial p}{\partial x_{i}}+\frac{1}{R e} \frac{\partial^{2} u_{i}}{\partial x_{j} \partial x_{j}}+R i \theta \delta_{i 3}+\delta_{i 1}, \\
& \frac{\partial \theta}{\partial t}+u_{j} \frac{\partial \theta}{\partial x_{j}}=\frac{1}{\operatorname{PrRe}} \frac{\partial^{2} \theta}{\partial x_{j} \partial x_{j}},
\end{aligned}
$$

where $R e, R i$, and $P r$ are reference the Reynolds, Richardson, and Prandtl numbers. Here, we assume constant background (reference) temperature $\theta_{0}$ in hydrostatic balance with background pressure. The variable $p$ is the pressure fluctuation from the background pressure. Density and temperature are related through the linearized equation of state for an ideal gas. ${ }^{2}$ In practice, we use unit reference values $u_{0}=1, h=1$, and $F=1$, so $u_{i}$ and $\theta$ can be interpreted as dimensional variables.

Wall scale nondimensional parameters are defined as

$$
R e_{\tau}=\frac{u_{\tau} h}{v}, \quad R i_{\tau}=\frac{g}{\theta_{0}} F \frac{h^{2}}{u_{\tau}^{2}},
$$

where friction velocity $\boldsymbol{u}_{\tau}=\left(\tau_{w} / \rho_{0}\right)^{\frac{1}{2}}, \rho_{0}$ is the reference density, and $g$ is the gravitational acceleration. Here, $\tau_{w}$ is the surface shear stress. The imposed uniform horizontal pressure gradient drives flow in the $x$ direction and forces the mean flow to balance the mean wall shear stress (averaged over the horizontal plane) and maintain the flow from deceleration in the streamwise direction. Thus, the time dependant values of $R i_{\tau}$ and $R e_{\tau}$ will converge to their corresponding reference values $R i$ and $R e$ as the flow approaches stationarity.

The bulk Richardson and Reynolds numbers are defined by

$$
R e_{b}=\frac{U_{b} h}{v}, \quad R i_{b}=\frac{g}{\theta_{0}} F \frac{h^{2}}{U_{b}^{2}},
$$

where $U_{b}=\frac{1}{h} \int \bar{u} d z$ is the bulk velocity, and $\overline{(~)}$ denotes averaging over horizontal planes at each $z$. Note that because the vertical gradient of temperature is specified at the lower surface, $R i_{\tau}$ is the parameter that determines cooling rate and therefore the stratification regime. Bulk and friction Richardson numbers are related through $R i_{b}=R i_{\tau} u_{\tau}^{2} / U_{b}^{2}$.

The boundary conditions are

$$
\begin{aligned}
& z=0: \quad u=v=w=0, \quad \frac{\partial \theta}{\partial z}=1, \\
& z=h: \quad \frac{\partial u}{\partial z}=\frac{\partial v}{\partial z}=w=\frac{\partial \theta}{\partial z}=0 .
\end{aligned}
$$


This choice of no heat flux at the upper boundary excludes heat entrainment from above and provides a framework to focus on the effect of surface cooling alone on wall generated turbulence. Although this choice leads to a constant rate of change in mean temperature, ${ }^{21}$ it will be shown that the mean velocity, mean temperature gradient, and fluctuating quantities reach equilibrium after sufficient time.

Two dimensionless scaling quantities, which are called wall units and frequently used in the analysis of wall-bounded flows, are defined as ${ }^{36,37}$

$$
z^{+}=\frac{z u_{\tau}}{v}, u^{+}=\frac{\bar{u}}{u_{\tau}} .
$$

Throughout the paper, superscript ${ }^{+}$denotes nondimensionalization by near wall scaling parameters $\left(u_{\tau}^{0}, v\right)$ (e.g., $\left.z^{+}=z u_{\tau}^{0} / v\right)$ and superscript ${ }^{o}$ represents nondimensionalization by outer layer scaling parameters $\left(u_{\tau}^{0}, h\right)\left(\right.$ e.g., $\left.t^{o}=t u_{\tau}^{0} / h\right)$, where $u_{\tau}^{0}$ refers to the initial value of the friction velocity (i.e., friction velocity of the neutral case).

It is well-known that different layers exist in neutrally stratified wall turbulence. These layers are classified as the viscous sublayer, buffer layer, logarithmic layer, and outer layer. ${ }^{37-39}$ With wall unit parameters, one can obtain universal profiles for mean velocity at a certain distance from the wall regardless of the details of the boundary layer. ${ }^{36}$ Collectively, the viscous sublayer, buffer layer, and logarithmic layer are referred to as the inner layer in this study. The outer layer is the outermost part of the boundary layer where viscosity has an insignificant effect on the mean and turbulent quantities. In the outer layer, the boundary layer thickness $\delta$ or channel flow half height is the length scale of the eddies.

\section{NUMERICAL APPROACH}

The numerical model used for this study is Hercules, which is a scalable open-source DNS solver that solves the OBA equation for a channel flow configuration. ${ }^{24}$ Minor modifications to include different type of surface temperature boundary conditions have been implemented in the code. Hercules has been validated for a wide range of Reynolds and Richardson numbers in a previous study ${ }^{24}$ for unstratified channel flow at $R e_{\tau}=180,395,590$ and for a stratified channel flow at $R e_{\tau}=180$ for Richardson numbers $R i_{\tau}=0,18$, 120,480 .

The governing equations are discretized using the Fourier spectral method in the horizontal plane with periodic boundary conditions and a second-order central finite difference scheme in the vertical direction. ${ }^{25}$ In horizontal directions, we employ a uniform mesh, colocated variables and use the Fourier transform to calculate derivatives. ${ }^{40}$ The two-thirds de-aliasing technique has been used to prevent aliasing error from energy transfer to unresolved wavenumbers. $^{41}$

In the wall-normal direction, a second order finite-difference scheme is used and the nonlinear terms are treated in skewsymmetric form to reduce the amplitude of aliasing errors ${ }^{42}$ for derivatives in the vertical direction. The mesh is Cartesian and nonuniform in the vertical direction. A vertically staggered grid is employed, in which the vertical velocity is located at cell faces and the horizontal velocity, pressure, and scalars are defined at cell centers. The staggering is done to remedy the problem of pressure-velocity decoupling and checkerboard oscillation of pressure. ${ }^{43}$ Linear interpolation is used to map variables between the half-grid and full-grid points. ${ }^{24,25}$

Hyperbolic tangent stretching is used in the $z$-direction to condense grid points near the solid boundaries to resolve the near-wall small-scale structures, with level $i$ at ${ }^{24,25}$

$$
z_{i}=h-\frac{h}{\tanh (2 \alpha)} \tanh \left(\frac{2 \alpha}{1-N_{z}}\left(i-N_{z}\right)\right),
$$

where $\alpha=1.1$ is a parameter to control the stretching and $N_{z}$ is the number of grid points in the vertical direction.

\section{A. Overview of simulations}

In this study, we considered one neutral and five stratified simulations with $R i$ up to 2800 . A summary table of the simulations is presented in Table I. Case C1 refers to the neutral case in which temperature is a passive scalar and buoyancy frequency

$$
N^{2}(z)=\frac{g}{\theta_{0}} \frac{\mathrm{d} \bar{\theta}}{\mathrm{d} z}
$$

is zero everywhere. Fields from the stationary state of the neutral case are used to initialize other simulations. The Prandtl number is set to unity in all cases.

Simulations are performed on a computational domain $L_{x} / h$ $=2 \pi, L_{y} / h=\pi$ with resolution $N_{x} \times N_{y} \times N_{z}=768 \times 768 \times 384$ for a moderate Reynolds number $R e=560$ which correspond to wall unit grid spacings $\Delta x^{+}=4.6, \Delta y^{+}=2.3$, and $\Delta z^{+} \in[0.08-3.3]$. The domain size and physical parameters are comparable to the case S00-S12 in the work of Flores and Riley ${ }^{7}$ but approximately three times higher spatial resolution in the horizontal directions and two times higher spatial resolution in the vertical direction are used to analyze small scale features of strongly stable wall turbulence. This resolution is comparable to the work of Vreman and Kuerten ${ }^{44}$ which used high resolution to study statistics of velocity derivatives. The time step is equal to $\Delta t=0.0002$ in $\mathrm{C} 1$ and $\mathrm{C} 2$ and $\Delta t$ $=0.00015$ for the other cases. Fields are output with at interval $t_{s}$, where $t_{s} u_{\tau} / h \approx[0.02-0.1]$. This output frequency corresponds to $t_{s} \approx[1-8] t_{\eta}$, where $t_{\eta}$ is the Kolmogorov time scale ${ }^{45}$ obtained using minimum values of the domain averaged viscous dissipation throughout simulation time history.

Although the size of the computational domain considered here is small, it is large enough to contain the widest flow structure

TABLE I. Main simulations parameters. In the last column, $t_{f}$ is the final time of the simulation. $R e$ and $R i$ are reference values for friction Reynolds $\left(R e_{\tau}\right)$ and Richardson numbers $\left(R i_{\tau}\right)$.

\begin{tabular}{lcccl}
\hline \hline Case & $R e$ & $R i$ & $h / L_{M O}$ & $t_{f} u_{\tau}^{0} / h$ \\
\hline C1 & 560 & 0 & 0 & 53.2 \\
C2 & 560 & 560 & 0.41 & 48.5 \\
C3 & 560 & 697 & 0.51 & 47.7 \\
C4 & 560 & 833 & 0.61 & 55.19 \\
C5 & 560 & 1120 & 0.82 & 62.6 \\
C6 & 560 & 2800 & 2.05 & 30.9 \\
\hline \hline
\end{tabular}


associated with at least one ejection and corresponding sweep, and therefore, it allows maintenance of "healthy" turbulence in the sense of producing correct mean flow behavior and one-point statistics when compared to simulations with larger domains. ${ }^{46,47}$ In addition, it has recently been shown that for channel flow simulations with passive heat transfer, this domain size accurately predicts the evolution of turbulent heat flux and RMS values of fluctuating temperature. $^{48}$

\section{RESULTS}

\section{A. Overview}

In this section, we first categorize the different phases of the wall turbulence response to surface cooling. Then, we further classify stratification regimes based on differences in the feedback process for different cooling rates. It will be shown that there is a critical cooling rate at which turbulence becomes patchy and beyond which there is total suppression of turbulence. The mechanism for these feedback processes is also discussed.

Figures 2(a)-2(d) show time series of friction and bulk Reynolds and Richardson numbers. Figures 2(e) and 2(f) show time series of domain integrated $k$ and $K$, where $k=\overline{u_{i}^{\prime} u_{i}^{\prime}} / 2$ is turbulent kinetic energy (TKE) and $K=\overline{u_{i} u_{i}} / 2$ is mean kinetic energy (MKE). Prime denotes fluctuation from horizontal average. After stratification is introduced at $t^{o}=0$, the friction velocity initially decreases, which leads to reduced wall shear stress. This reduction in shear stress with increasing stratification is consistent with field observations of stably stratified ABLs ${ }^{49,50}$ and laboratory experiments of SBLs. ${ }^{51}$ Consequently, the bulk velocity and bulk Reynolds number increase due to flow acceleration. The evolution of the friction Reynolds number exhibits three different phases: turbulence decay, turbulence recovery, and a quasistationary state. The evolution of domain integrated TKE also shows similar trend [Fig. 2(f)].

Turbulence decay starts from the introduction of stratification at $t^{o}=0$ and lasts up to $t^{o} \approx 4-6$ in all cases, where TKE reaches its minimum through the cooling process. The recovery phase starts at the time associated with the TKE minimum. The duration of the recovery phase depends on $R i$, where higher $R i$ requires more time for recovery. For example, while for case $\mathrm{C} 2$, the recovery phase continues up to $t^{o} \approx 20$, for $\mathrm{C} 5$, it lasts until $t^{o} \approx 45$. This difference is consistent with the time scale of the flow adjustment $L_{M O} / u_{\tau}$ as suggested in Ref. 7 . However, the recovery phase is different for the lower $R i_{\tau}$ cases (C2 and C3) compared to the higher $R i_{\tau}$ cases ( $\mathrm{C} 4$ and $\mathrm{C} 5)$. In the former cases recovery involves a monotonic increase in TKE to a quasistationary state, while in the latter case, there is an overshoot of TKE before the turbulence approaches quasistationarity.

Similar to the plots of $R e_{\tau}$ in Fig. 2(b), for C2 and C3, $k$ in Fig. 2(f) decays and then recovers until it reaches quasistationarity, while $K$ monotonically increases until becoming quasisteady, as shown in Fig. 2(e). By contrast, in C4 and C5, $K$ increases initially, reaches a maximum, and then decreases to a quasistationary state, while $k$ decays until $t \approx 4$ and then recovers and reaches a maximum at $t^{o} \approx 21$ for case C4 and $t^{o} \approx 24$ for case C5. After this time, the bulk flow decelerates and $k$ reaches quasiequilibrium. While the minimum $k$ in the decay phase decreases with increasing stratification, the $k$ maxima in the recovery phase for cases $\mathrm{C} 4$ and C5 cases increases with stratification.
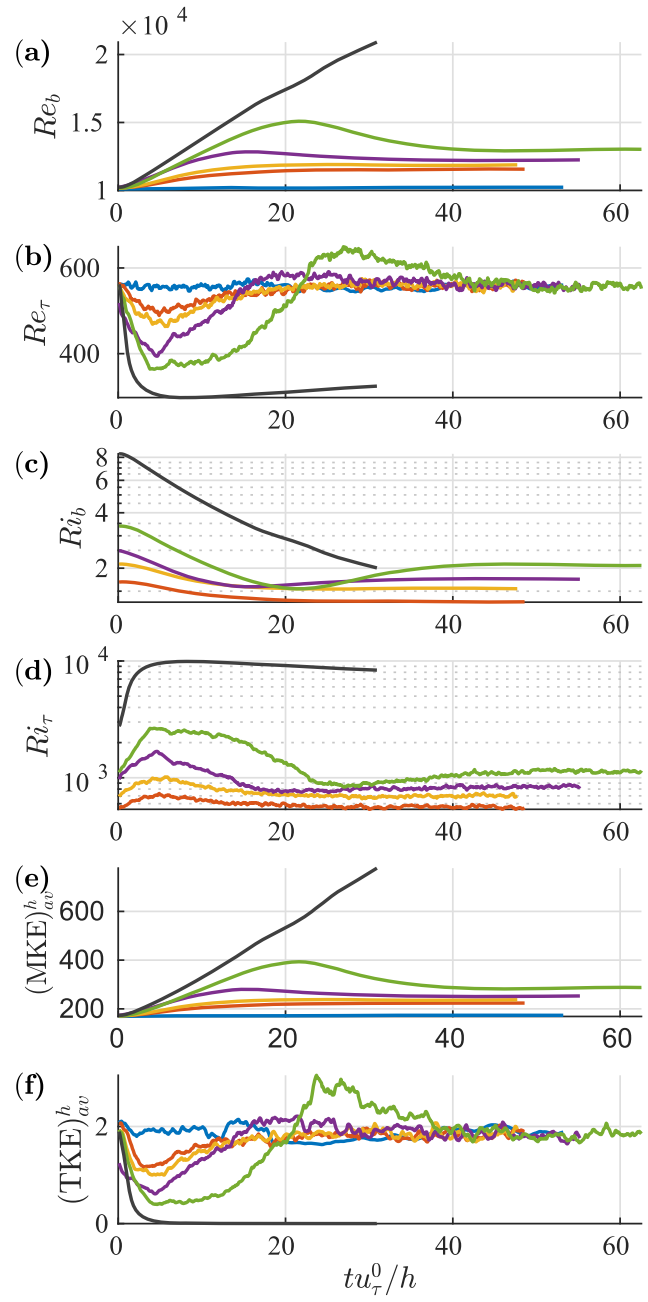

FIG. 2. Time series of (a) bulk Reynolds number, (b) friction Reynolds number, (c) bulk Richardson number, (d) friction Richardson number, (e) domain integrated MKE, and (f) domain integrated TKE. The blue, red, yellow, magenta, green, and black lines are for $\mathrm{C} 1, \mathrm{C} 2, \mathrm{C} 3, \mathrm{C} 4, \mathrm{C} 5$, and $\mathrm{C} 6$, respectively.

Time evolution of the friction and bulk Richardson numbers are shown in Figs. 2(c) and 2(d). $R i_{b}$ and $R i_{\tau}$ are inversely proportional to the bulk and wall scale kinetic energy, respectively. These plots show a trend opposite to $R e_{b}, R e_{\tau}$ suggesting that $R i_{b}, R i_{\tau}$ correlates with the conversion of kinetic energy to potential energy in mean and fluctuating fields, respectively.

The most stratified case C6 in Fig. 2 shows laminarization, as indicated by the sudden drop in $R e_{\tau}$, until $t^{o} \approx 15$ (as will be shown further below). We did not continue this case to achieve quasistationarity.

These results support earlier studies ${ }^{7,22,26}$ that have used $h / L_{M O}$ to categorize the dependence of SBL turbulence on the cooling rate. For $h / L_{M O}<0.6$ (cases C2 and C3), turbulence is affected by stratification but continuously exists, similar to weakly stratified cases as in earlier works. ${ }^{20,21}$ For $h / L_{M O} \geq 0.6$ (cases C4, C5), in the recovery state, TKE achieves a larger peak than in the neutral case. For 


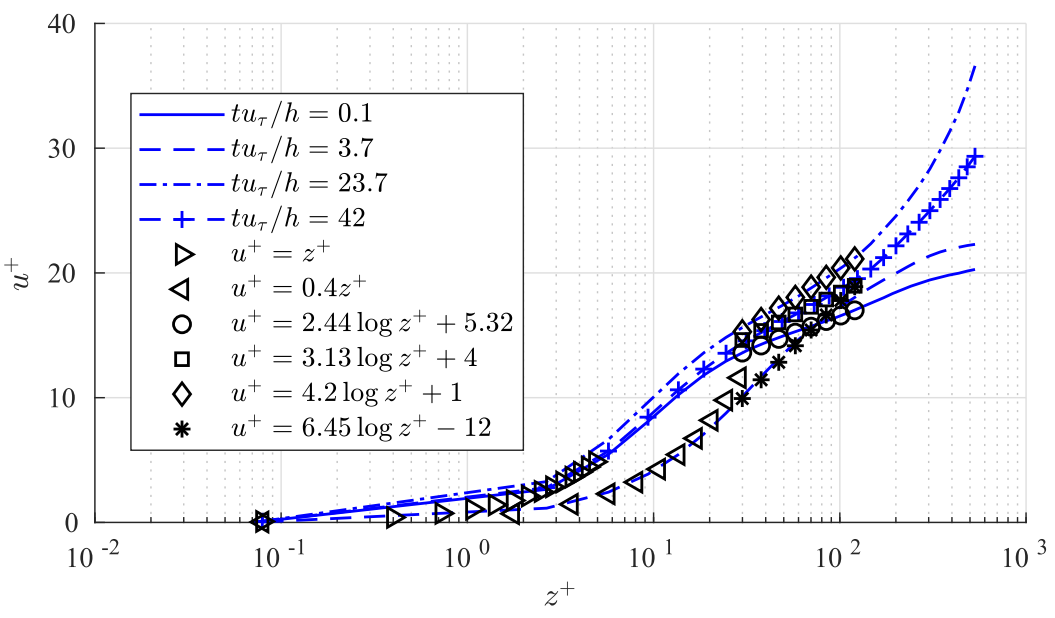

FIG. 3. Mean velocity profiles for case $\mathrm{C} 5$ at early time $t^{0}$ $=0.1$ (solid), time of maximum decay $t^{0}=3.7$ (dashed), end of recovery $t^{\circ}=23.7$ (dashed-dotted), and stationarity $t^{\circ}$ $=42$ (dash-cross sign). $h / L_{M O} \gtrsim 1$ (case C6), the energy-containing modes are strongly affected by stable stratification and laminarization is the dominant feature of the decay phase. However, there is uncertainty in the literature about exact criteria for turbulence collapse for stably stratified wall-bounded turbulence. The $h / L_{M O}>1.2$ criteria was reported ${ }^{22,26}$ in earlier studies for turbulence collapse. Nevertheless, this criteria might be affected by the Reynolds number, ${ }^{7,26}$ computational domain size, ${ }^{23}$ and also initial conditions. ${ }^{7,28,29}$

The main focus of the present paper is case C5. However, we partly discuss other cases and, in particular, C6. The C2-C4 simulations are mainly presented to exhibit the overall effects of weaker stratification (compared to C5 and C6) on different phases of the cooling process.

\section{B. Identifying inner and outer regions}

To study the response of wall turbulence to surface cooling in the decay and recovery phases, we now identify how different layers (viscous sublayer, buffer layer, logarithmic, and outer layer) respond to different cooling rates.

In C5, because of the initially strong suppression of TKE, the wall-normal distances over which these layers are defined can be different from the neutral case. To see this discrepancy, consider Fig. 3, where $u^{+}$are shown at different times during the surface cooling process for case C5. It is clear that the decay phase changes the viscous sublayer such that parabolic behavior of this layer extends up to $z^{+}=15$ and the buffer layer shrinks. However, log-linear behavior up to $30 \lesssim z^{+} \lesssim 100$ is still observed but with different constants in the log-linear profile at different times. By using mixing length theory and linear variability of length scales with respect to $z$ within the logarithmic layer, ${ }^{52}$ the turbulence production in the logarithmic region is closely related to the slope of the log-linear profile. However, while the slope is not significantly different at different times in Fig. 3, the constant in the log-linear profiles varies substantially, suggesting that this coefficient is set by the details of other layers and not the logarithmic layer itself. Changes of slope of the loglinear profile between two successive times are smaller than changes of the additive constant of the log-linear profile between two successive times. The slope increases monotonically, while the additive constant undergoes sharp changes. The mean velocity profile in the viscous sublayer and buffer layer at $t^{o}=23.7$ is similar at $t^{\circ}=42$, but the outer layer behavior is different. This difference in outer layer behavior suggests that the additive constant in the log-linear profile is set by the details of the outer layer, similar to the unstratified logarithmic layer.

To avoid the complication of the evolution of the viscous and buffer layers as the surface cools, we focus on the inner layer, where $z^{+} \leq 100$, and outer layer, where $z^{+}>100$. This criterion separates the inner and outer regions at all times in Fig. 3 and is also valid for cases C2-C4 (no shown). With this splitting, we can analyze the differences in how the inner and outer regions respond to surface cooling.

\section{Inner and outer layer response to surface cooling}

Figure 4 shows the integrated TKE rate of change over the inner and outer layers scaled with their initial values in each of those layers for the decay and recovery phases. In this figure, $\left\langle\mathrm{d} k_{a v} / \mathrm{d} t\right\rangle$ is
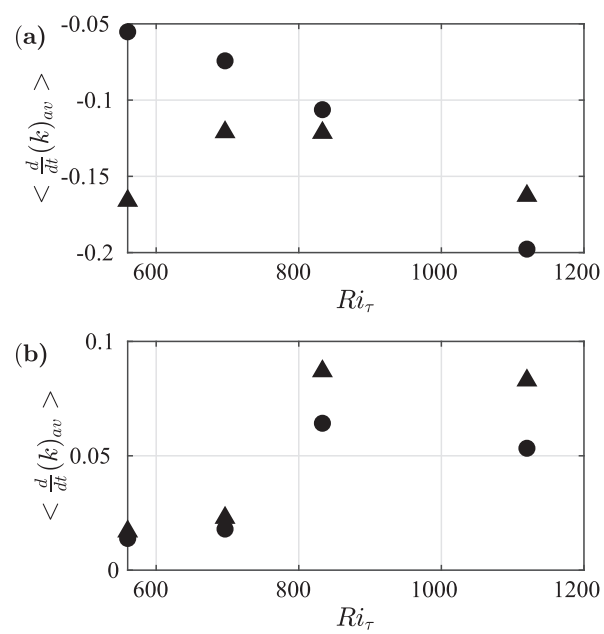

FIG. 4. Time-averaged decay rate of scaled (with respect to its initial value for each layer and each $R i_{\tau}$ ) integrated TKE in the inner (circles) and outer (triangles) layers, averaged over the (a) decay phase and (b) recovery phase. 
the average TKE rate of change, where \langle\rangle means time average of the quantity over the desired period. The subscript $a v$ refers to layer over which integration of TKE is performed (i.e., inner or outer).

In the decay phase [Fig. 4(a)], it can be seen that for C2 and $\mathrm{C} 3$, the outer layer has the largest decay rate. However, as stratification increases, the difference between the decay rate in the inner and outer layers becomes smaller. Indeed, for C5, the inner layer has the largest decay rate, suggesting a possible collapse of near-wall turbulence. We will show later that for this case, near wall turbulence does collapses temporarily and the flow becomes patchy. In the recovery phase [Fig. 4(b)], the outer layer has the largest recovery rate, but the difference between recovery rates of the inner and outer layers increases with increasing $R i_{\tau}$, suggesting that there is a time delay between the recovery of the inner and outer layers. Generally, the decay rate is faster than the recovery rate for the cases considered here.

\section{Feedback process in C5 and C6}

Turbulence in cases $\mathrm{C} 2-\mathrm{C} 4$ is continuously sustained. However, in $\mathrm{C} 5$, it becomes patchy soon after the beginning of surface cooling. As shown in Fig. 5, this patchy turbulence exists for about 10 eddy turnover times $\left(t^{o} \approx 10\right)$ in the recovery phase until turbulence is eventually fully revived. Our main interest in this paper is to study features of the turbulence in the strongly stratified case C5. We present a detailed look at this case in this section. For comparison, we also consider the most strongly stratified case C6.

Horizontal snapshots of the streamwise velocity fluctuations for case C5 are shown at four vertical levels in Fig. 5 for different times during the cooling process. Shortly after the start of surface cooling at $t^{o} \approx 0.1$, near-wall streaks spread over the full horizontal plane [Figs. 5(a)-5(c)]. Moving upward from the inner to outer layer, the streamwise aligned structures become more isotropic, as
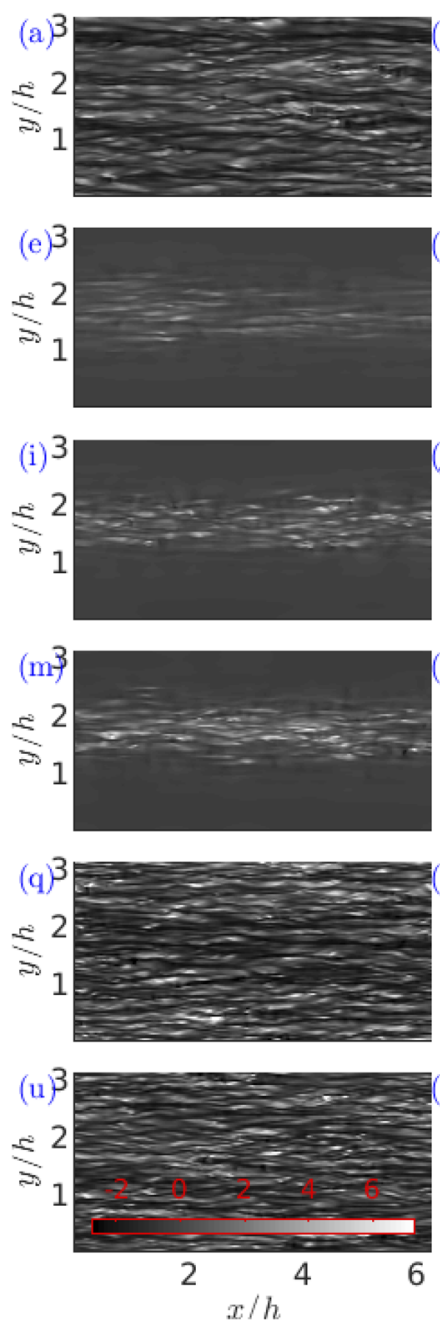
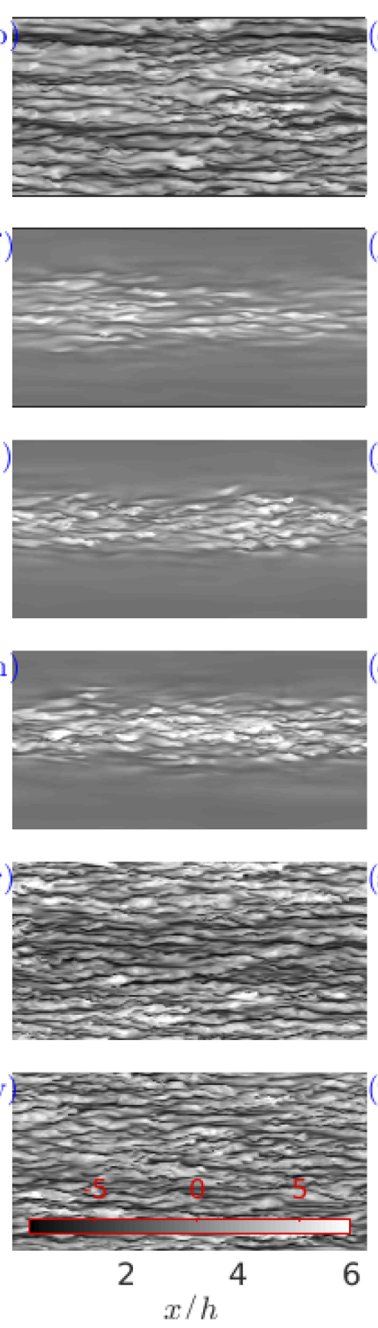
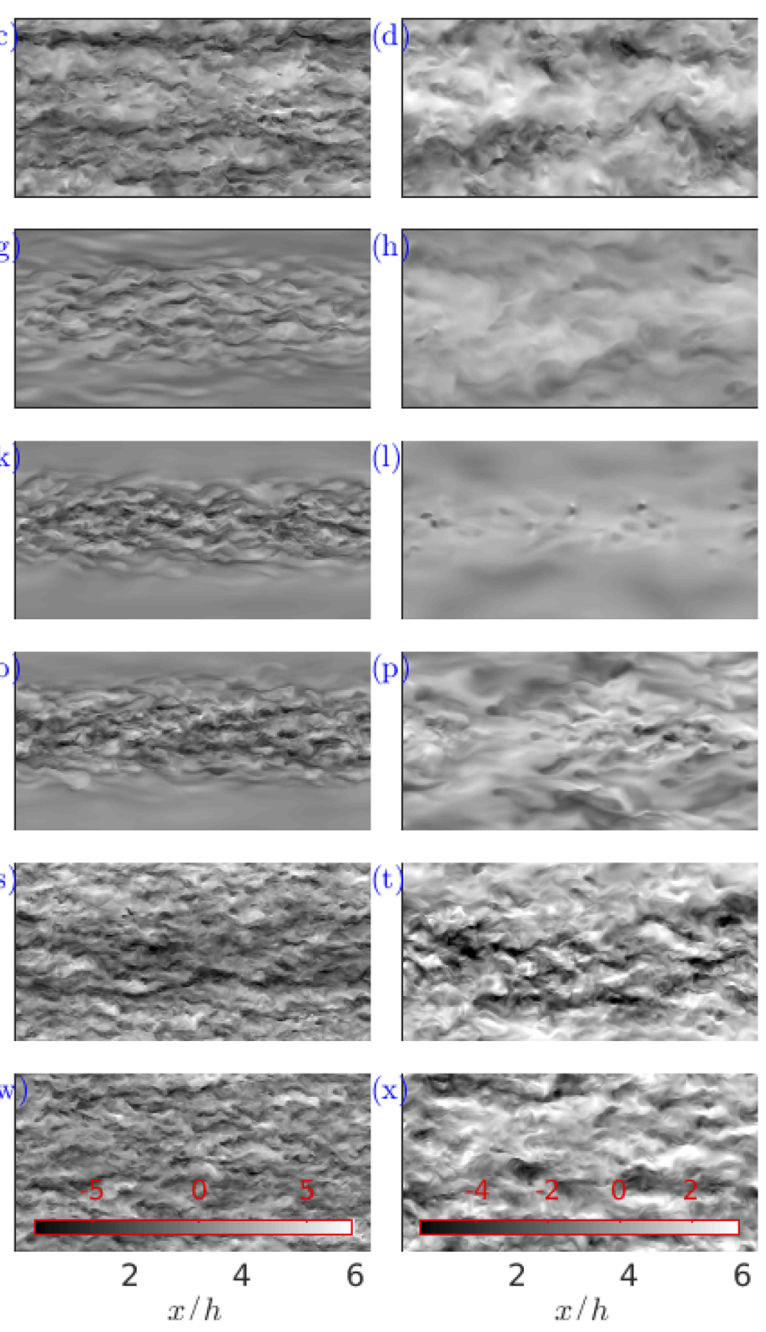

FIG. 5. Fluctuating streamwise velocity $u^{\prime+}$ at different layers and different times for C5. Each column shows a different level; from left to right, these are $z^{+}=3.5[(a)$, (e), (i), $(\mathrm{m}),(\mathrm{q})$, and $(\mathrm{u})], 15[(\mathrm{~b}),(\mathrm{f}),(\mathrm{j}),(\mathrm{n}),(\mathrm{r})$, and $(\mathrm{v})], 70[(\mathrm{c}),(\mathrm{g}),(\mathrm{k}),(\mathrm{o}),(\mathrm{s})$, and $(\mathrm{w})]$, and $300[(\mathrm{~d}),(\mathrm{h}),(\mathrm{l}),(\mathrm{p}),(\mathrm{t})$, and $(\mathrm{x})]$. Each row shows a different time; from top to bottom, these are $t^{0}=0.1[(\mathrm{a})-(\mathrm{d})], 3.7[(\mathrm{e})-(\mathrm{h})], 8.7[(\mathrm{i})-(\mathrm{l})], 14.7[(\mathrm{~m})-(\mathrm{p})], 23.7[(\mathrm{q})-(\mathrm{t})]$, and $29.7[(\mathrm{u})-(\mathrm{x})]$. Colorbars on each layer are based on $u^{\prime+}$ for different layers at $t^{\circ}=0.1$. 
shown in Fig. 5(d). During the decay phase, turbulence decays in all layers, and, as shown in Figs. $5(\mathrm{e})-5(\mathrm{~g})$ at $t^{o}=3.7$, the structures in the inner layer, and in particular near-wall streaks, disappear in some regions. Interestingly, laminar-turbulence coexistence is observed in Figs. $5(\mathrm{e})-5(\mathrm{~g}), 5(\mathrm{i})-5(\mathrm{k})$, and $5(\mathrm{~m})-5(\mathrm{o})$, with a turbulent strip of spanwise width $\lambda_{y} \approx 1.5 h$ in the viscous sublayer and buffer layer. Turbulence is suppressed outside the strip. The turbulent strip becomes narrower in the early part of recovery at $t^{o}=8.7,14$ [Figs. 5(i), 5(j), 5(m), and 5(n)], decreasing to $\lambda_{y} \approx$ $h$. Meanwhile, the fluctuations within the turbulent strip become stronger [e.g., streak velocities become stronger in Figs. 5(i) and 5(m) compared to Fig. 5(e)]. The logarithmic layer also follows the trend of the near-wall regions as it becomes patchy. However, it has a wider turbulent strip.

As cooling of the surface continues, the patchiness eventually extends to the outer region at $t^{o}=14.7$ as shown in Fig. 5(p), which strongly supports the necessity of inner layer production in sustaining outer layer turbulence. However, patchiness in the outer layer disappears after a few eddy turn over times, as shown in Fig. 5(x).

During the recovery in Figs. 5(i)-5(t), inner layer TKE builds up although some patchiness still exists. The inner layer structures are fully recovered by $t^{o}=23.7$. It will be discussed below that the quiet regions in the neighborhood of the turbulent strip in Fig. 5, which are often referred to as a laminar region, ${ }^{7,8}$ actually appear to consist of layered turbulence with flat structures.

Profiles of $k$ at different times in the cooling process are shown in Fig. 6. The decay, recovery, and approach to quasiequilibrium can be seen in this plot. At early times $t^{o} \lesssim 3.7$, the TKE maximum shifts upward to $z^{+} \approx 30$ as a result of the shrinking buffer layer and boundary layer growth, as shown by the mean velocity and temperature gradients in Fig. 7. At later times, the maximum TKE occurs around

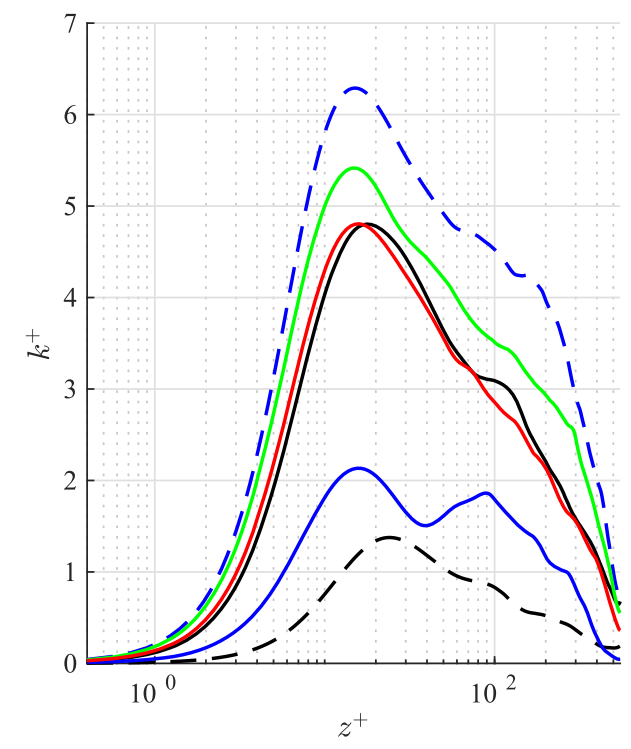

FIG. 6. Instantaneous TKE for $\mathrm{C} 5$ at different times. The solid black line is for $t^{\circ}$ $=0.1$, the dashed black line is for $t^{0}=3.7$, the solid blue line is for $t^{0}=14.7$, the dashed blue line is for $t^{0}=23.7$, the green line is for $t^{0}=29.7$, and the red line is for $t^{0}=41.9$.
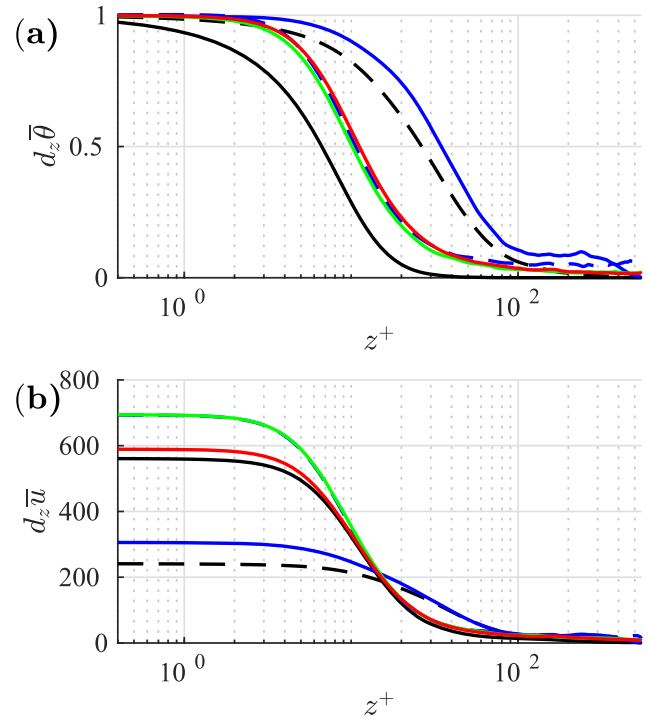

FIG. 7. (a) Instantaneous mean temperature and (b) velocity gradient for $\mathrm{C} 5$ at different times. The solid black line is for $t^{0}=0.1$, the dashed black line is for $t^{\circ}$ $=3.7$, the solid blue line is for $t^{0}=14.7$, the dashed blue line is for $t^{\circ}=23.7$, the green line is for $t^{0}=29.7$, and the red line is for $t^{\circ}=41.9$.

$z^{+}=15$, suggesting that the buffer layer is rebuilding. During the recovery phase, when TKE in the buffer layer starts to recover, TKE in the outer layer with $z^{+} \gtrsim 300$ is still decreasing. This sequencing strongly suggests that there is a delayed recovery in the outer layer, which uses energy that has been produced earlier in the buffer layer during the decay phase. This delay in recovery is consistent with the discussion given in Sec. IV C.

The boundary layer thickness starts growing during the decay phase, and it continues growing until $t^{o} \approx 13$ in the recovery phase, as shown in Fig. 7. Mean shear at the wall and in the region $z^{+} \lesssim 20$ is built up during the recovery phase. Therefore, this region may be responsible for turbulence production when sufficiently large tangential Reynolds stress is available. Both mean shear and mean temperature gradient acquire quasiequilibrium at $t^{0} \approx 42$.

Profiles of the different contributions to the TKE budget are presented in Fig. 8 (see the Appendix). Except for the viscous sublayer, where viscous diffusion has large values, the main balance in the TKE budget at all times is between production and dissipation. The peak of buoyancy destruction at $z^{+} \approx 100$ is an order of magnitude smaller than the peak of production and dissipation. In the lower part of the viscous sublayer, the wall impermeability prevents turbulent production when $z^{+} \lesssim 2$ at all times. Except for early times when peak production shifts upward as a result of boundary layer growth, dissipation dominates the inner layer outside of the buffer layer $5 \lesssim z^{+} \lesssim 30$, where production has larger values. Above $z^{+} \approx 100$, production and dissipation are balanced. Production and dissipation are weakened during the decay phase $0.1 \lesssim t^{0} \lesssim 3.7$, suggesting that the appearance of the patches results from a lack of production rather than an excess in dissipation. The impact of lack of production in turbulence collapse is also supported by experimental studies of SBLs. ${ }^{5}$ 

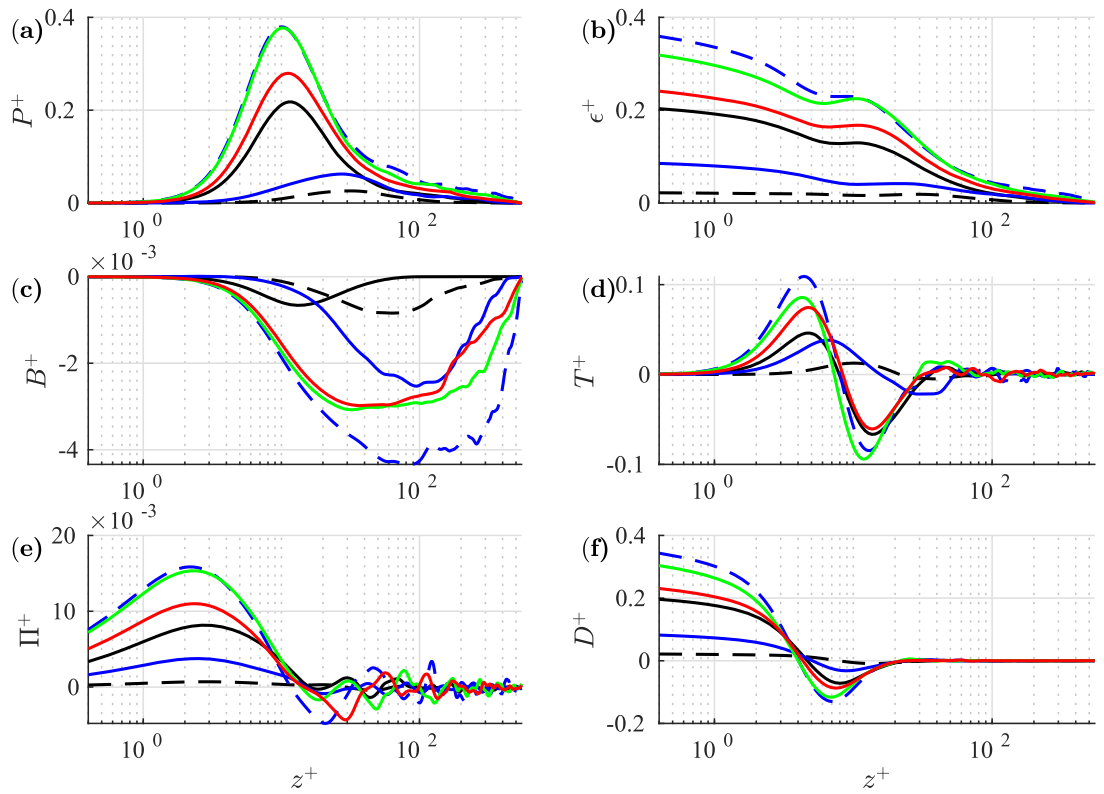

FIG. 8. Different contributions to the evolution of TKE for C5 at different times: (a) production, (b) dissipation, (c) buoyancy destruction, (d) turbulent transport, (e) pressure-work, and (f) viscous diffusion. The solid black line is for $t^{0}=0.1$, the dashed black line is for $t^{0}=3.7$, the solid blue line is for $t^{0}=14.7$, the dashed blue line is for $t^{0}=23.7$, the green line is for $t^{\circ}=29.7$, and the red line is for $t^{\circ}$ $=41.9$.
Furthermore, tangential Reynolds stresses are suppressed in the decay phase [Fig. 9(a)] as a result of the damping effect of stable stratification, which leads to damping of vertical velocity fluctuations. Therefore, ejection and sweep events are hindered. This
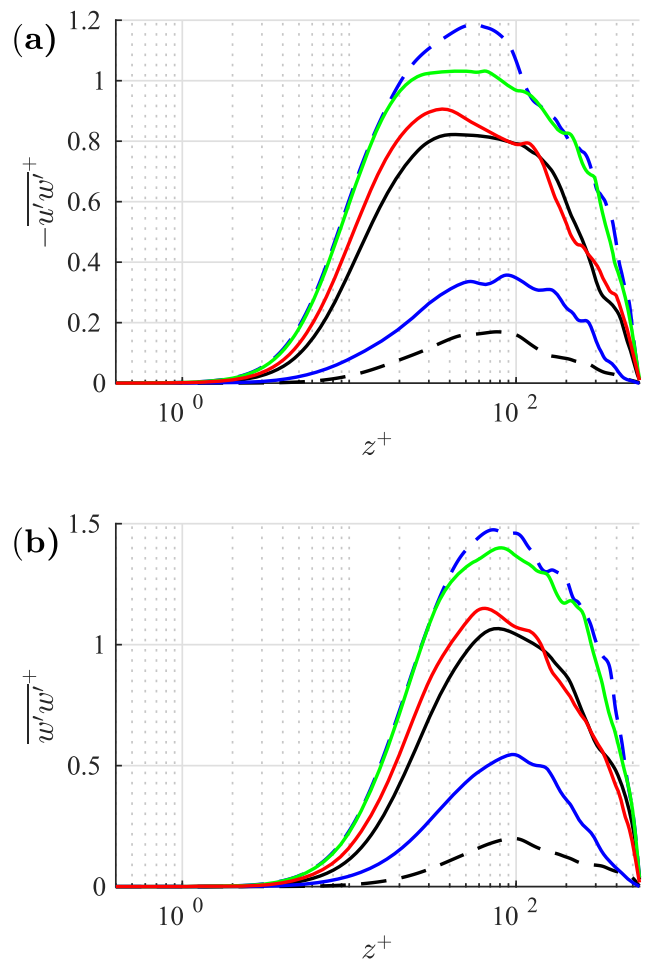

FIG. 9. Instantaneous (a) $-\overline{u^{\prime} w^{\prime}}$ and (b) $\overline{w^{\prime} w^{\prime}}$ for $\mathrm{C} 5$ at different times. The black lines correspond to the decay phase. The solid black line is for $t^{0}=0.1$, the dashed black line is for $t^{0}=3.7$, the solid blue line is for $t^{0}=14.7$, the dashed blue line is for $t^{0}=23.7$, the green line is for $t^{0}=29.7$ and the red line is for $t^{0}=41.9$. interruption in momentum transfer can also be seen in $T^{+}$, Fig. 8(d), where upward TKE transfer is almost fully suppressed and downward TKE transfer is significantly reduced. This is expected since once fluid in the near-wall region becomes heavier as a result of surface cooling, more energy is required for vertical motions. The buoyancy frequency has larger values as we approach the wall due to the thermal boundary condition (Fig. 7), and therefore fluid closer to the wall feels a stronger restoring force. Pressure-work has its largest value in the lower part of the viscous sublayer at all times.

Figure 10 shows streamwise velocity fluctuations for case C6. This figure reveals that for the strongest surface cooling, while turbulence in the inner region is damped in Figs. 10(e)-10(g) and $10(\mathrm{i})-10(\mathrm{k})$, the outer layer [Figs. $10(\mathrm{~h})$ and 10(1)] still has active turbulence (although the intensity of the fluctuations is strongly reduced). The surviving streaks at time $t^{\circ}=3.7$ in the buffer region [Fig. 10(f)] are of size $\lambda_{x} \approx L_{x}$, suggesting they are associated with the largest modes that the computational domain can handle. Structures similar to the streaks in the buffer region (with higher intensity) exist in the logarithmic layer for this time. The buffer and logarithmic layers also shows analogous large scale features. ${ }^{53,54}$ In this case, the near wall streak intensities are an order of magnitude smaller than those in case C5 at the same time. The inner and outer layers are almost decoupled. The diffusive nature of the inner region for case C6 is also clear. Continuing surface cooling leads to complete removal of quasistreamwise structures and continuous decrease in intensity of fluctuations. By time $t^{o}=14$, outer layer turbulence is also damped.

\section{E. Feedback mechanism in C5 and C6}

In this section, we discuss possible mechanisms that may explain the response of the flow to the surface cooling described in Sec. IV D. We first visualize the change in flow structures with cooling. We use the Q-criterion, which is the second invariant of the velocity gradient tensor, which is defined as 

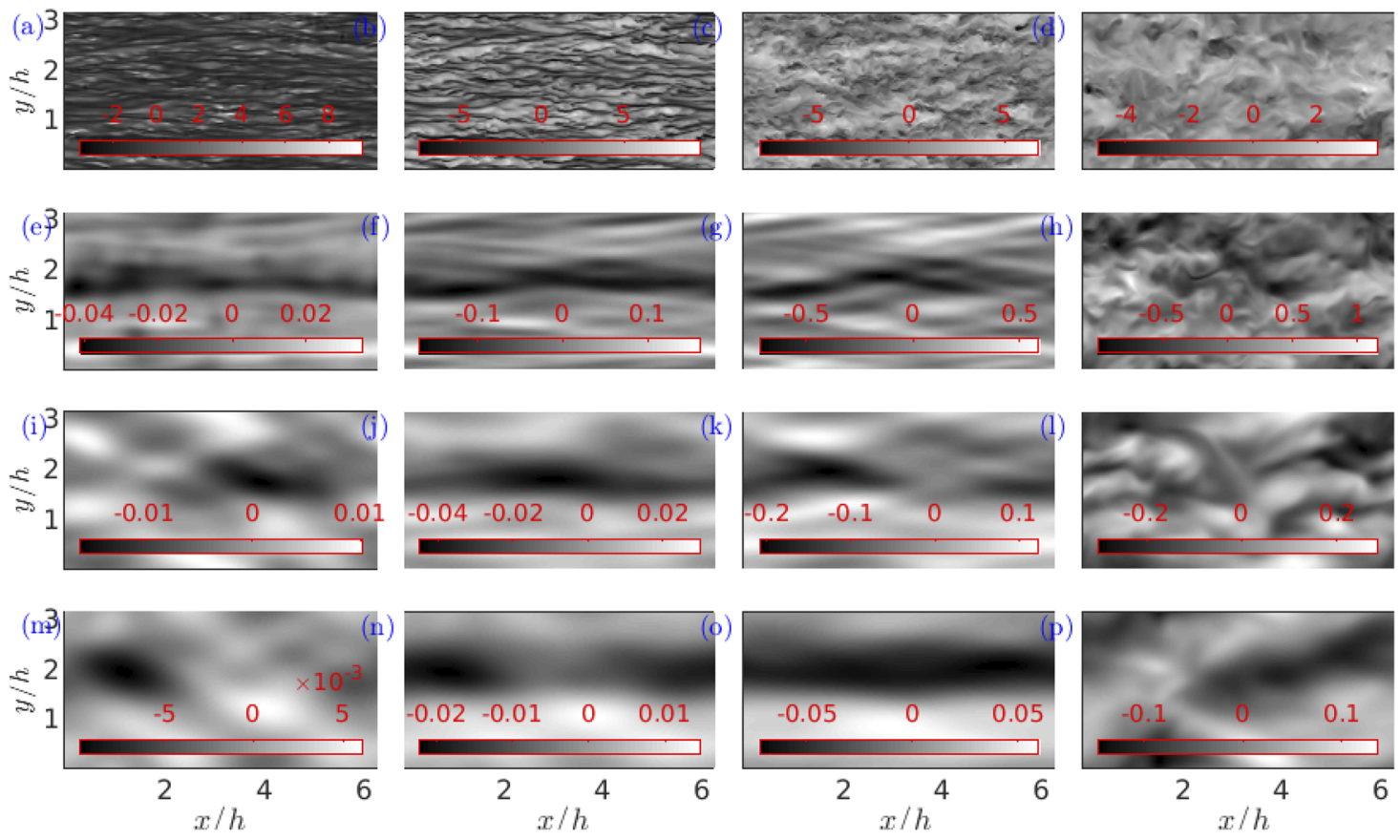

FIG. 10. Fluctuation streamwise velocity $u^{\prime+}$ at different layers and different times for $\mathrm{C} 6$. Each column shows a different level; from left to right, these are $z^{+}=3.5[(a)$, (e), $(\mathrm{i})$, and $(\mathrm{m})], 15[(\mathrm{~b}),(\mathrm{f}),(\mathrm{j})$, and $(\mathrm{n})], 70[(\mathrm{c}),(\mathrm{g}),(\mathrm{k})$, and $(\mathrm{o})]$, and $420[(\mathrm{~d}),(\mathrm{h}),(\mathrm{l})$, and $(\mathrm{p})]$. Each row shows a different time; from top to bottom, these are $t^{0}=0.1[(\mathrm{a})-(\mathrm{d})]$, $3.7[(\mathrm{e})-(\mathrm{h})], 8.7[(\mathrm{i})-(\mathrm{l})]$, and $14.7[(\mathrm{~m})-(\mathrm{p})]$.

$$
Q=-\frac{1}{2} \frac{\partial u_{i}}{\partial x_{j}} \frac{\partial u_{j}}{\partial x_{i}} .
$$

Figure 11 shows $Q$ at $t^{o}=0.1$. At this early time, turbulence spans whole cross section and the near wall region $\left(z^{+} \leq 30\right)$ is dominated by quasistreamwise vortical structures. Lifted-up vortices from these quasistreamwise vortical structures are also present and are surrounded by other incoherent structures aloft. These nearwall structures contribute to sweep and ejection events associated with pulling high-momentum fluid from upper levels and pushing

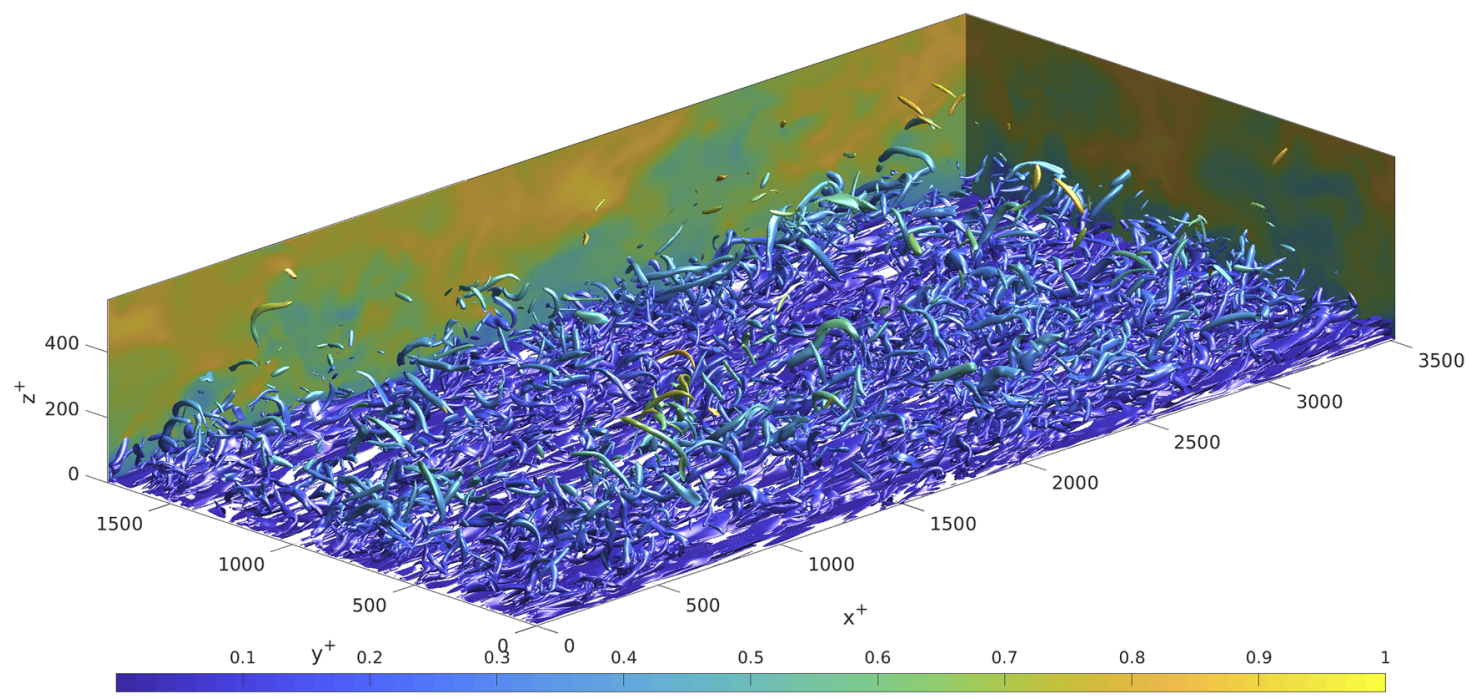

FIG. 11. Visualization of instantaneous scaled $Q^{\prime+}$ at time $t^{0}=0.1$ as an indication of vortical structures for case $C 5$ colored by distance from the wall. Cross-sectional slices are total kinetic energy in streamwise and spanwise planes. Both $Q^{\prime+}$ and total kinetic energy are scaled with their maximum values. Isosurfaces of scaled $Q^{\prime+}$ are plotted at the level of 0.01 . The colorbar is based on values of scaled total kinetic energy. 
low-momentum fluid to upper levels, creating velocity streaks and maintaining turbulence. However, as shown in Figs. 5 and 10, stable stratification can change the organization, population, and intensity of these structures.

Figure 12 shows $Q$ at a later time $t^{o}=11$, in the early stage of the recovery phase. Contour plots of the total kinetic energy are also included to indicate energy transfer. The modulation of vortical structures caused by stable stratification is clear. The quasistreamwise vortical structures are absent in the regions outside of the turbulent strip, which we refer to as quiet zones. Turbulence is concentrated in the strip, which comprises numerous quasistreamwise vortices and hairpinlike structures. ${ }^{56,57}$ However, the quantity of lifted and detached vortices is considerably lower in comparison with $t^{o}=0.1$, even in the turbulent strip which can explain the reduction in turbulence production from its initial value. This modulation can also be seen in the contour plots of the total kinetic energy in Fig. 12, where vertical transport of lower to higher momentum from the inner to outer layer is significantly altered. Boundary layer growth up to $z^{+} \approx 300$ can be seen from these contour plots along with shear instabilities at the top of the boundary layer.

An important question is how turbulence recovers from the patchy state in C5. Different scenarios may be considered for this recovery: it could be due to spread of the turbulent strip in the spanwise direction as a result of built-up of shear and tangential Reynolds stress as the front of the turbulent strip destabilizes the neighboring quiet region ${ }^{58}$ leading to spreading of turbulence in the spanwise direction, and/or it could be excitation from large scale eddies aloft that can trigger transition and cause recovery. Hairpinlike vortices are observed in Fig. 12 at $y^{+} \gtrsim 1500$ in the near-wall region for $x^{+} \gtrsim 2000$ and $x^{+} \approx 3500$. This hairpin vortex is 200 wall units in the spanwise direction away from the turbulent strip, which is consistent with the scenario based on excitation from the boundary layer top. On the other hand, if the recovery is independent of fluctuations at the boundary layer top, then turbulence in the quiet region should be recovered in the absence of those fluctuations.

To test this hypothesis, we perform two experiments in which we remove the fluctuations at certain heights from the right-hand side of the governing equation by replacing the terms in Eqs. (3) and (4) with their horizontal average. The fluctuations are removed above $z^{+}=200$ in the first experiment and $z^{+}=300$ in the second. Both experiments are initialized using fields from C5 at time $t u_{\tau} / h=3.7$ associated with maximum decay. Time series of domain integrated MKE and TKE are shown in Fig. 13 for these two experiments. These plots reveal that while MKE is essentially the same in both experiments, the presence of fluctuations at $z^{+} \gtrsim 300$ is important for recovery from localized patch such as a turbulent strip. This finding supports the hypothesis that recovery in the quiet regions is triggered by large structures in the outer layer rather than spreading of the strip.

Thus, the recovery process in C5 can be understood as follows: Once turbulence reaches maximum decay in the inner layer, energy-containing eddies in the outer layer still use TKE that has been produced in the buffer region at previous times (which had higher TKE) due to delay in the decay of the inner and outer layers. These outer layer eddies can control decay of inner layer TKE from further fall-off through interaction of inner and outer layers where large scale eddies of the size of $\lambda_{z}^{+} \approx 300(\gtrsim h / 2)$ in the outer layer can penetrate down close to the wall and stir the flow (in the sense of Townsend's wall attached eddies hypothesis ${ }^{39}$ ).

Active eddies in the turbulent strip in the buffer layer will continuously maintain turbulence in the log and outer layers. However, since these eddies lose a significant portion of their TKE during the decay phase, the outer layer will not be maintained sufficiently and will experience decay at a later time.

Excitation of inner layer turbulence by outer layer sweeping in the decay phase helps to reinvigorate the regeneration cycle of

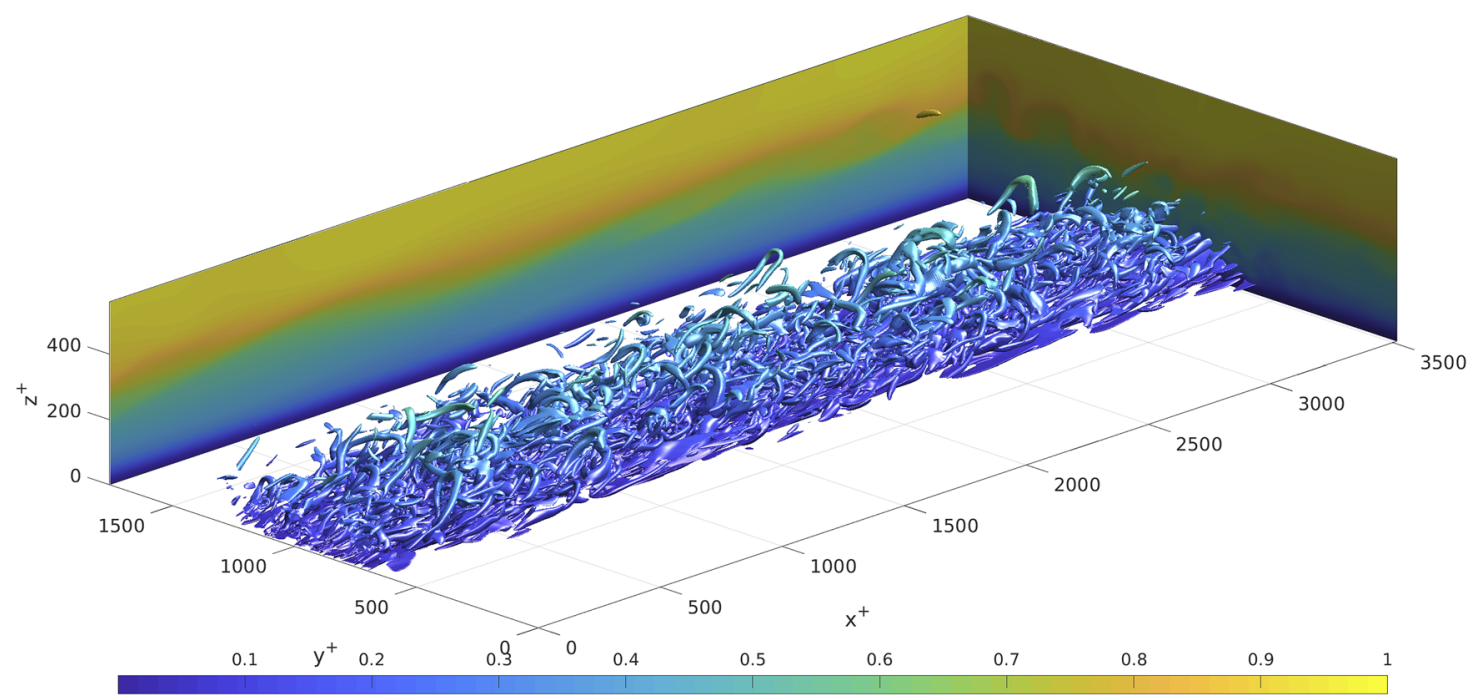

FIG. 12. Visualization of instantaneous scaled $Q^{\prime+}$ criterion at time $t^{0}=11$ as an indication vortical structures for case C5 colored by distance from the wall. Cross-sectional slice plots are total kinetic energy in streamwise and spanwise planes. Both $Q^{\prime+}$ and total kinetic energy are scaled with their maximum values. Isosurfaces of scaled $Q^{\prime+}$ are plotted at the level of 0.01 . The colorbar is based on values of scaled total kinetic energy. 

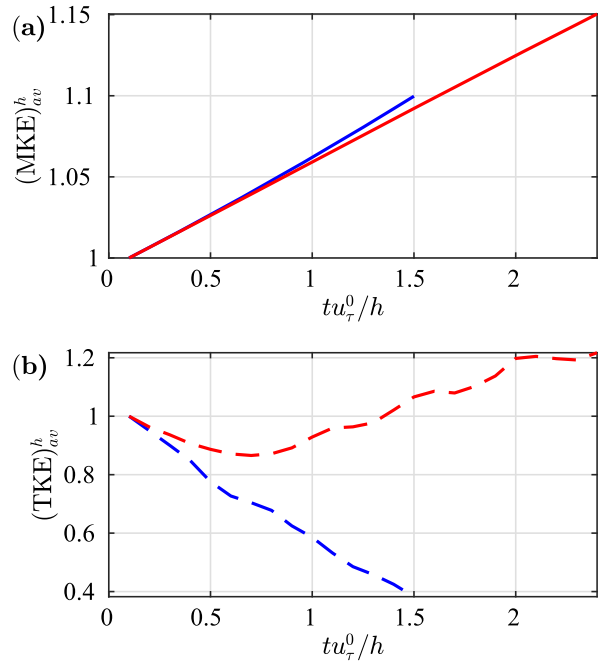

FIG. 13. Time series of (a) domain averaged MKE and (b) domain averaged TKE for $\mathrm{C} 5$ experiments where fluctuations are removed above $z^{+}=200$ (blue lines) and $z^{+}=300$ (red lines). MKE and TKE have been scaled by their initial values. Time zero here corresponds to $t u_{\tau} / h=3.7$ in Fig. 2 .

near-wall turbulence. ${ }^{60}$ Then, streaks in the viscous sublayer and buffer layer reappear, reactivating ejection and sweep events in quiet regions near the wall. Eventually, turbulence spans the whole channel width and fully recovers. While TKE is building up in the inner layer, a smaller amount of production from the inner layer is needed for outer layer recovery, and therefore the outer layer recovers faster due to fact that the effect of viscosity is less significant there.

The most stratified case C6 is different. Figure 14 shows $Q$ for C6 at a later time $t^{o}=23.7$. It can be seen that the cooling process leads to a collapse of the vertical scale in the vortical structures in the inner region and leads to the formation of quasiflat structures. The flat structures are also observed in the outer layer. As shown in Fig. 10, elongated streamwise structures are replaced by these flat pancakelike structures.

In the stably stratified $\mathrm{ABL}$, intermittences such as local patches of turbulence also exist under strongly stable stratification. ${ }^{3}$ A similar recovery process to what we have discussed in this section has also been suggested in field studies of the stable ABL. These studies suggest that the patchiness in strongly stable ABLs could be interrupted through the intrusion of shear instabilities from the boundary layer aloft into the stable surface layer. ${ }^{61,62}$

\section{F. Nature of quiet zones in C5}

In this section, we consider the characteristics of quiet regions adjacent to the turbulent strip that appears during the cooling process in C5. To do so, we investigate the spectra of the streamwise velocity fluctuations.

The one-dimensional longitudinal spectra of streamwise velocity, averaged over $y$ at $z^{+}=15$, are shown in Fig. 15. Spectra are averaged in $y$ over regions that become quiet during the decay phase and recovery phase. As expected, initially, the spectrum shows an approximate $k_{x}^{-5 / 3}$ behavior over $10 \lesssim k_{x} h \lesssim 100$, where $k_{x}$ is the streamwise wave number. During decay as shown in Fig. 15(b), the spectrum become steeper over these wave numbers as turbulence is suppressed. The slope of the spectrum increases in the recovery phase, as shown in Figs. 15(c) and 15(d). However, for about 5 eddy turnover times, the slope of the spectrum is approximately -5 , as shown in Figs. 15(c) and 15(d), similar to viscously coupled stratified turbulence. ${ }^{63,64}$ In the logarithmic region, the steepening of the spectrum is weaker compared to the near-wall region and the slope of spectra becomes approximately -3, as shown in Figs. 16(a)-16(f). The smallest wavenumbers, where $k_{x} h<10$, present $k_{x}^{-1}$ behavior

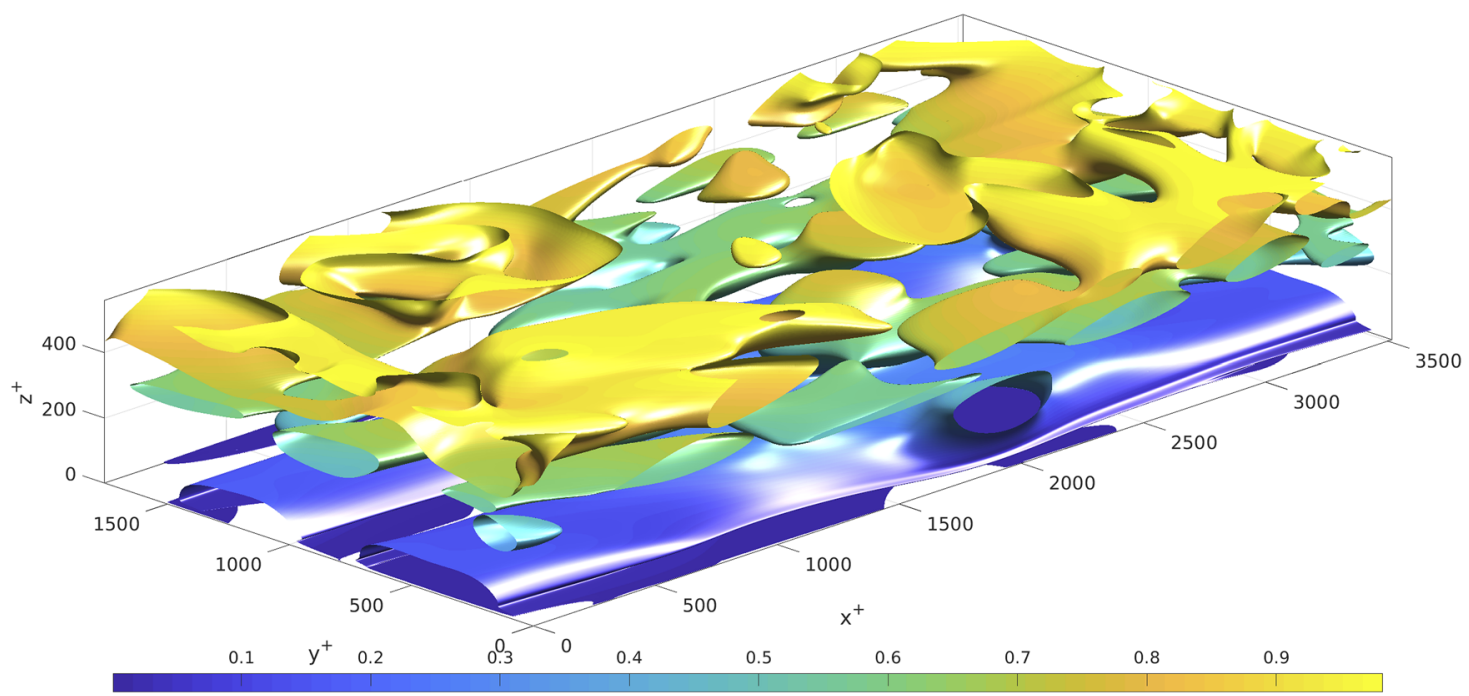

FIG. 14. Visualization of instantaneous $Q^{\prime+}$ criterion at time $t^{0}=23.7$ as an indication vortical structures for case $C 6$ colored by distance from the wall. $Q^{\prime+}$ is scaled with their maximum values. Isosurfaces of scaled $Q^{\prime+}$ are plotted at the level of 0.01 . The colorbar is based on distance from the wall. 

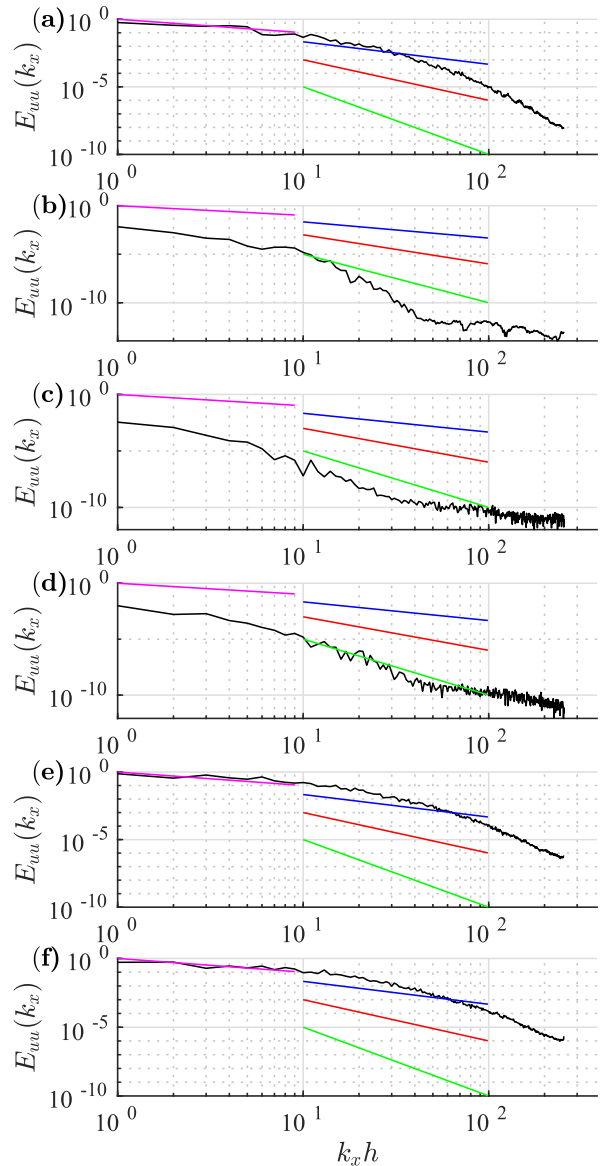

FIG. 15. Longitudinal spectra of streamwise kinetic energy $E_{u u}\left(k_{x}\right)$ at $z^{+}=15$ at different times in a subbox corresponding to a quiet region at maximum decay, which is $5 \pi / 6 \leq y / h \leq \pi$. Times correspond to (a) $t^{0}=0.1$, (b) $t^{0}=3.7$, (c) $t^{0}=8.7$, (d) $t^{0}=14$, (e) $t^{0}=23.7$, and (f) $t^{0}=32.9$, respectively. Magenta, blue, red, and green lines have slope of $-1,-5 / 3,-3$, and -5 , respectively.

initially [Figs. 15(a) and 16(a)] and again once turbulence is recovered [Figs. 15(e), 15(f), 16(e), and 16(f)].

The behavior of the spectra suggests that the quiet regions may consist of viscously layered pancake vortices, where vertical fluctuations are strongly suppressed by stable stratification and the vertical scale of the eddies is set by viscosity. ${ }^{64,65}$ In this regime, the Ozmidov scale is smaller than the Kolmogorov scale, where the Ozmidov scale is defined as $L_{o}=\left(\epsilon / N^{3}\right)^{1 / 2}$. The buoyancy Reynolds number, which is related to the ratio of Ozmidov to Kolmogorov scales, is defined as $^{66}$

$$
R e_{B}=\left(\frac{L_{o}}{\eta}\right)^{3 / 4}=\frac{\epsilon}{v N^{2}},
$$

where $\eta=\left(v^{3} / \epsilon\right)^{1 / 4}$ is the Kolmogorov scale. $R e_{B}$ can be used to detect regions of viscously coupled pancake vortices: regions where $R e_{B} \ll 1$ correspond to viscously layered vortices, and regions where $R e_{B} \gg 1$ contain overturning and small scale vortices. Plots of
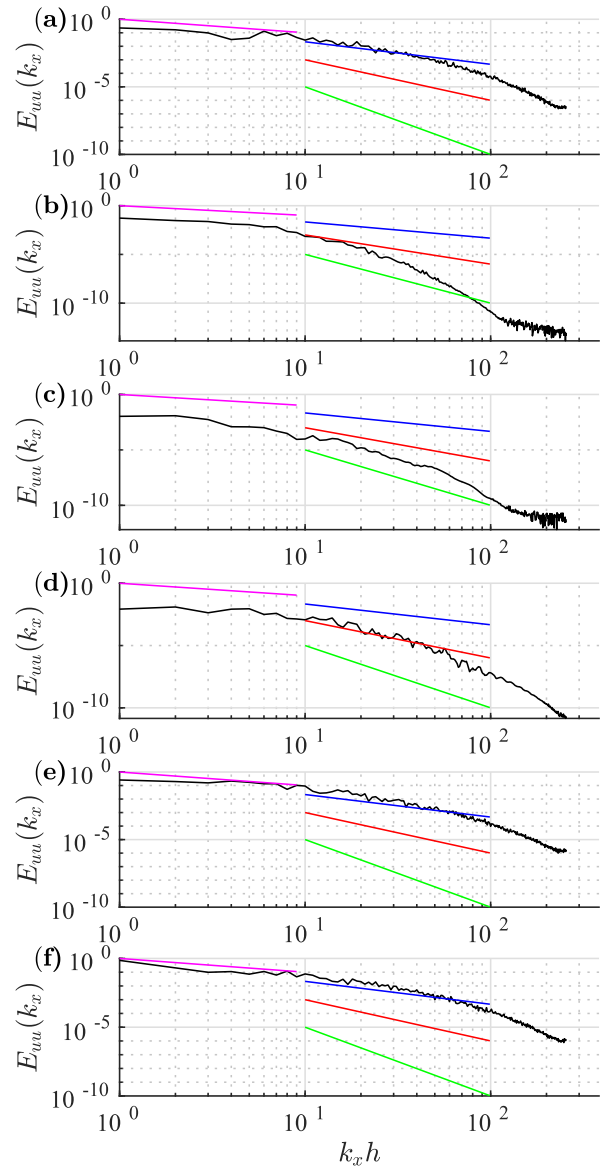

FIG. 16. Longitudinal spectra of streamwise kinetic energy $E_{u u}\left(k_{x}\right)$ at $z^{+}=70$ at different times in a subbox corresponding to a quiet region at maximum decay, which is $5 \pi / 6 \leq y / h \leq \pi$. Times correspond to (a) $t^{0}=0.1$, (b) $t^{\circ}=3.7$, (c) $t^{\circ}=8.7$, (d) $t^{\circ}=14$, (e) $t^{\circ}=23.7$, and (f) $t^{\circ}=32.9$, respectively. Blue, red, green, and cyan lines have slope of $-1,-5 / 3,-3$, and -5 , respectively.

horizontally averaged $R e_{B}$ in the quiet regions and the turbulent strip are shown in Fig. 17. At $t u_{\tau} / h=0.1$, these two regions have the same $R e_{B}$ due to spanwise homogeneity of the turbulence at this time. For both of these regions, $R e_{B}>1$ where $z^{+}>10$. At later times, until turbulence is fully recovered, $R e_{B}$ in the quiet region is smaller than $R e_{B}$ in the active region. During the decay phase as shown in Fig. 17(b), $R e_{B}$ in both regions is reduced. In the turbulent strip, $R e_{B}<1$ for up to $z^{+} \approx 60$, while in the quiet region, $R e_{B} \ll 1$ even up to $z^{+} \approx 200$. The steepening of the spectrum corresponds to a reduction in $R e_{B}$. When the slope of $E_{u u}\left(k_{x}\right)$ is close to -5 in the quiet region during recovery [Figs. 15(c) and 15 (d)], $R e_{B}$ in the same region is $O\left(10^{-4}\right)$, which is two orders of magnitude smaller than $R e_{B}$ in the turbulent strip. Once turbulence is fully recovered, $R e_{B}$ for these two regions become similar again.

\section{G. Effect of upper thermal boundary condition}

The results shown thus far were obtained using a neutral boundary condition for heat transfer from the upper boundary of 

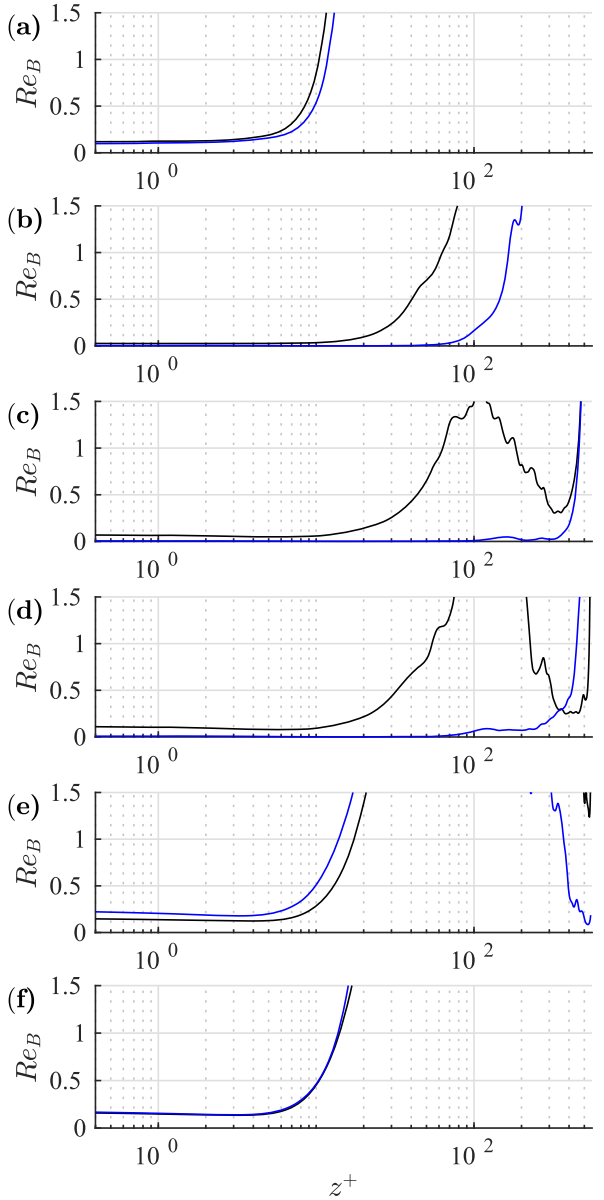

FIG. 17. Buoyancy Reynolds number computed in the quiet region (blue lines) where $5 \pi / 6 \leq y / h \leq \pi$ and active regions (black lines) $\pi / 3 \leq y / h \leq 2 \pi / 3$. Times correspond to (a) $t^{0}=0.1$, (b) $t^{0}=3.7$, (c) $t^{0}=8.7$, (d) $t^{0}=14$, (e) $t^{0}=23.7$, and (f) $t^{0}=32.9$, respectively.

the SBL. If heat entrainment from the upper boundary is permitted, it may affect the decay and recovery of turbulence since heating the SBL from the top leads to the formation of a capping inversion. ${ }^{28,6}$

In Ref. 7, a Dirichlet boundary condition for temperature was used at the upper boundary. In their $R i=1120$ simulation initialized from a neutral case, only the experiment perturbed with $5 \%$ higher-than-average TKE in the outer region could recover. Here, we observed that $\mathrm{C} 5$ can recover from partial collapse without perturbations to the initial condition. This suggests that the SBL upper boundary condition can play an important role when strong cooling from the bottom surface is imposed. To investigate this, we carried out another simulation with the initial condition and parameters identical to $\mathrm{C} 5$ but with a Dirichlet boundary condition $(\theta=0)$ at the upper boundary (case C5D). In Fig. 18(a), a time series of the domain-integrated TKE for this case is shown and compared to that from C5. Although heat entrainment from the upper boundary leads to a decrease in decay rate up to $t^{\circ} \approx 4$, the time of recovery in $\mathrm{C} 5$, the C5D case continues decaying after this time. This finding strongly
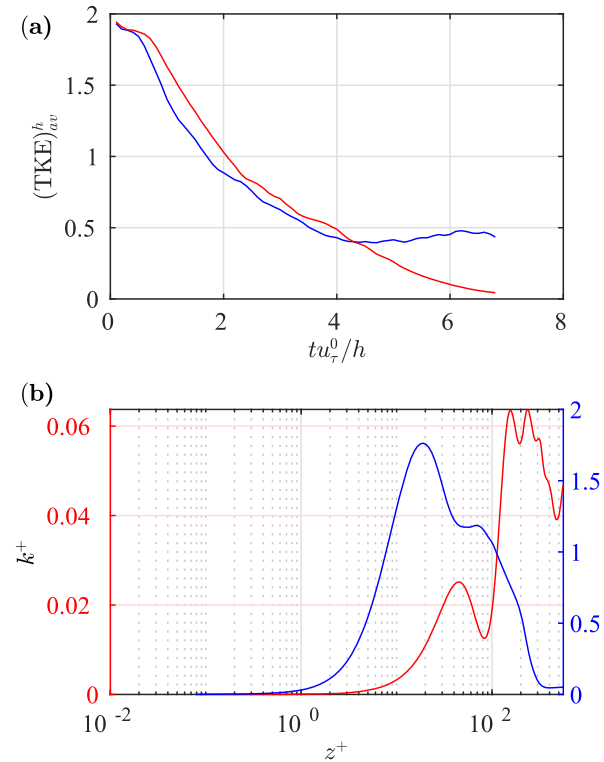

FIG. 18. Effects of the upper boundary condition on (a) domain integrated TKE and (b) TKE profile at $t^{\circ}=6.8$ for C5 (blue) and an analogous simulation (C5D) with Dirichlet boundary conditions at the upper boundary (red, with $\theta=0$ at $z=h$ ).

suggests that the impact of the upper boundary condition on outer layer dynamics influences recovery since (as shown earlier) recovery in C5 is strongly linked with outer layer large-scale structures. It should be pointed out that in $\mathrm{C} 5 \mathrm{D}$, turbulence become patchy as well shortly after introducing stratification. Nevertheless, by $t^{\circ} \approx 6.8$, near-wall streaks disappear in C5D as decay continues. For example, the value of TKE at $z^{+}=15$ becomes almost two orders of magnitude smaller than C5 at the same time, as shown in Fig. 18(b). The shift in TKE for C5D toward higher $z^{+}$is likely a result of laminarization, where wall shear is strongly reduced and the boundary layer cannot accommodate a buffer layer. In laminarization of the boundary layer, the buffer layer is not capable of sufficient turbulence production to overcome dissipation caused by viscosity. Thus, the energetic eddies are located farther from the wall since viscous dissipation is reduced moving from the wall toward the channel center.

\section{CONCLUSION}

In this paper, the response of wall turbulence to strong surface cooling was studied using high-resolution DNS. It was shown that the cooling process can be divided into different phases depending on the cooling rate. Turbulence first undergoes decay regardless of the imposed cooling rate. However, recovery largely depends on the cooling rate. For a weaker cooling rate, turbulence is ubiquitous at all vertical levels, consistent with previous studies. ${ }^{20,21}$ In this situation, TKE recovers to a level comparable to the neutral case. For stronger cooling rates, TKE reaches values during recovery that are higher than those in the neutral case. In these strongly stratified cases, turbulence may collapse partially with the SBL turbulence becoming patchy, or turbulence may collapse totally. 
For patchy turbulence $(R i=1120)$, it was shown that during decay, the viscous sublayer grows to $z^{+} \approx 15$ and the buffer region shrinks as the boundary layer grows. However, logarithmic behavior is observed up to $z^{+} \approx 100$ at all times, with slope and additive constants that depend on time.

For $R i=1120$ (case C5), a thorough analysis of the TKE budget confirms that the dominant balance is between production and dissipation at all times. It was shown the appearance of patchiness is due to a significant reduction in turbulent production (due to direct impact of stable stratification on tangential Reynolds stress) and not excess dissipation. This reduction in production is then linked to a decrease in the population and intensity of quasistreamwise vortical structures. During decay and part of the recovery phase, a quiet region appears in the near-wall region with width between $[1-1.5] h$.

It was shown that the quiet regions outside of the turbulent strip actually resemble viscously layered stratified turbulence, with layered vortices, suppressed vertical momentum flux, and $R e_{B} \ll 1$. In these regions, longitudinal spectra of streamwise velocity shows $k_{x}^{-5}$ behavior in the buffer layer and $k_{x}^{-3}$ in the logarithmic layer.

In the most stratified case with $R i=2800$ (case C6), the inner layer turbulence is completely suppressed and the outer layer turbulence decays subsequently, leading to the formation of flat structures in the near-wall region and pancakelike vortices in the outer layer.

The recovery of turbulence in $\mathrm{C} 5$ is discussed in detail. One might say for this Richardson number $(R i=1120)$, the autonomous cycle of near-wall turbulence, where turbulence in the near-wall region can autonomously sustain itself without intervention of eddies from the core region, ${ }^{68}$ is partially or totally hindered in the decay phase by the action of strongly stable stratification. In C5, however, the outer layer large scales of size $\lambda_{z}^{+} \gtrsim 300$ have enough intensity to excite the partially damped turbulence in the inner layer, which can restart the inner layer regeneration cycle. ${ }^{60,68}$ In C6 $(R i=2800)$, this autonomous cycle of near-wall turbulence is suppressed completely as near-wall streaks are damped soon after starting the cooling process.

The effect of the higher Reynolds number, Richardson numbers, and larger domain size on the characteristics of the patchy state, the evolution of TKE and vorticity, and possible turbulence recovery will be the subject of future work. These simulations can improve our understanding of the more realistic SBL, in particular, once turbulence becomes intermittent for strongly stable stratification. Moreover, in future work, it could be interesting to explore non-OBA effects on strongly stable stratified wall-bounded flow by solving the Navier-Stokes equations under the low Mach number approximation.

\section{ACKNOWLEDGMENTS}

The authors are grateful to Dr. Ping He, who provided valuable assistance during the code development stage. This research was enabled in part by support provided by the Shared Hierarchical Academic Research Computing Network (SHARCNET), Compute/Calcul Canada, the Natural Sciences and Engineering Research Council of Canada (K.A.S.: Grant No. RGPIN-418344-2012; and M.L.W.: Grant No. RGPIN-386456-2015), and the Canadian Foundation for Innovation.

\section{APPENDIX: TKE BUDGET}

The evolution of TKE in the SBL is governed $b^{20}$

$$
\frac{\partial k}{\partial t}+\bar{u}_{j} \frac{\partial k}{\partial x_{j}}=P+T+\Pi+D+\epsilon+B
$$

where

$$
\begin{aligned}
& P=-\overline{u_{i}^{\prime} u_{j}^{\prime}} \frac{\partial \bar{u}_{i}}{\partial x_{j}}, \\
& T=-\frac{\partial \overline{u_{i}^{\prime} u_{i}^{\prime} u_{j}^{\prime}}}{\partial x_{j}}, \\
& \Pi=-\frac{\partial \overline{p^{\prime} u_{i}^{\prime}}}{\partial x_{i}}, \\
& D=\frac{1}{R e} \frac{\partial^{2} k}{\partial x_{j} \partial x_{j}}, \\
& \epsilon=-\frac{2}{R e} \frac{\partial u_{i}^{\prime}}{\partial x_{j}} \frac{\partial u_{i}^{\prime}}{\partial x_{j}}, \\
& B=R i \overline{u_{i}^{\prime} \theta^{\prime}} \delta_{i 3},
\end{aligned}
$$

are production, turbulent transport, pressure-transport (pressure work), viscous diffusion, viscous dissipation, and buoyant destruction, respectively.

\section{REFERENCES}

${ }^{1}$ R. B. Stull, An Introduction to Boundary Layer Meteorology (Springer Science \& Business Media, 2012), Vol. 13.

${ }^{2}$ J. C. Wyngaard, Turbulence in the Atmosphere (Cambridge University Press, 2010).

${ }^{3}$ L. Mahrt, "Stably stratified atmospheric boundary layers," Annu. Rev. Fluid Mech. 46, 23-45 (2014)

${ }^{4}$ F. Zonta and A. Soldati, "Stably-stratified wall-bounded turbulence," Appl. Mech. Rev. 70, 040801 (2018).

${ }^{5}$ V. Armenio and S. Sarkar, "An investigation of stably stratified turbulent channel flow using large-eddy simulation,” J. Fluid Mech. 459, 1-42 (2002).

${ }^{6}$ F. Karimpour and S. K. Venayagamoorthy, "On turbulent mixing in stably stratified wall-bounded flows," Phys. Fluids 27, 046603 (2015).

${ }^{7}$ O. Flores and J. J. Riley, "Analysis of turbulence collapse in the stably stratified surface layer using direct numerical simulation," Boundary-Layer Meteorol. 139, 241-259 (2011).

${ }^{8}$ G. Brethouwer, Y. Duguet, and P. Schlatter, “Turbulent-laminar coexistence in wall flows with Coriolis, buoyancy or Lorentz forces," J. Fluid Mech. 704, 137-172 (2012).

${ }^{9}$ L. Ran, Z. Deng, X. Xu, P. Yan, W. Lin, Y. Wang, P. Tian, P. Wang, W. Pan, and D. Lu, "Vertical profiles of black carbon measured by a micro-aethalometer in summer in the North China Plain," Atmos. Chem. Phys. 16, 10441-10454 (2016).

${ }^{10}$ A. J. Ding, T. Wang, V. Thouret, J.-P. Cammas, and P. Nédélec, “Tropospheric ozone climatology over Beijing: Analysis of aircraft data from the MOZAIC program," Atmos. Chem. Phys. 8, 1-13 (2008).

${ }^{11}$ Z. Li, J. Guo, A. Ding, H. Liao, J. Liu, Y. Sun, T. Wang, H. Xue, H. Zhang, and B. Zhu, "Aerosol and boundary-layer interactions and impact on air quality," Nat. Sci. Rev. 4, 810-833 (2017).

${ }^{12}$ J. Cloern, "Temporal dynamics and ecological significance of salinity stratification in an estuary (South San-Francisco Bay, USA)," Oceanol. Acta 7, 137-141 (1984). 
${ }^{13}$ R. Adrian, D. O. Hessen, T. Blenckner, H. Hillebrand, S. Hilt, E. Jeppesen, D. M. Livingstone, and D. Trolle, "Environmental impacts-Lake ecosystems," in North Sea Region Climate Change Assessment, edited by M. Quante and F. Colijn (Springer International Publishing, Cham, 2016), pp. 315-340.

${ }^{14} \mathrm{M}$. Abkar and F. Porté-Agel, "The effect of free-atmosphere stratification on boundary-layer flow and power output from very large wind farms," Energies 6 , 2338-2361 (2013).

${ }^{15} \mathrm{~J}$. J. Riley and M.-P. Lelong, "Fluid motions in the presence of strong stable stratification," Annu. Rev. Fluid Mech. 32, 613-657 (2000).

${ }^{16} \mathrm{E}$. Lindborg, "The energy cascade in a strongly stratified fluid," J. Fluid Mech. 550, 207-242 (2006)

${ }^{17}$ C. J. Lang and M. L. Waite, "Scale-dependent anisotropy in forced stratified turbulence," Phys. Rev. Fluids 4, 044801 (2019).

${ }^{18}$ J. Jiménez, "The physics of wall turbulence," in Proceedings of the 20th IUPAP International Conference on Statistical Physics [Physica A 263, 252-262 (1999)].

${ }^{19}$ J. Jiménez, "Near-wall turbulence," Phys. Fluids 25, 101302 (2013).

${ }^{20}$ R. P. Garg, J. H. Ferziger, S. G. Monismith, and J. R. Koseff, "Stably stratified turbulent channel flows. I. stratification regimes and turbulence suppression mechanism," Phys. Fluids 12, 2569-2594 (2000).

${ }^{21} \mathrm{~J}$. R. Taylor, S. Sarkar, and V. Armenio, "Large eddy simulation of stably stratified open channel flow," Phys. Fluids 17, 116602 (2005).

${ }^{22}$ F. T. M. Nieuwstadt, "Direct numerical simulation of stable channel flow at large stability,” Boundary-Layer Meteorol. 116, 277-299 (2005).

${ }^{23}$ M. García-Villalba and J. C. del Álamo, "Turbulence modification by stable stratification in channel flow," Phys. Fluids 23, 045104 (2011).

${ }^{24} \mathrm{P}$. He, "A high order finite difference solver for massively parallel simulations of stably stratified turbulent channel flows," Comput. Fluids 127, 161-173 (2016).

${ }^{25}$ R. P. Garg, J. H. Ferziger, and S. G. Monismith, "Hybrid spectral finite difference simulations of stratified turbulent flows on distributed memory architectures," Int. J. Numer. Methods Fluids 24, 1129-1158 (1997).

${ }^{26}$ E. Deusebio, P. Schlatter, G. Brethouwer, and E. Lindborg, "Direct numerical simulations of stratified open channel flows," J. Phys.: Conf. Ser. 318, 022009 (2011).

${ }^{27} \mathrm{O}$. Flores and J. J. Riley, "Energy balance in stably-stratified, wall-bounded turbulence," in Mixing and Dispersion in Flows Dominated by Rotation and Buoyancy, edited by H. J. Clercx and G. F. Van Heijst (Springer International Publishing, Cham, 2018), pp. 89-99.

${ }^{28}$ J. M. M. Donda, I. G. S. van Hooijdonk, A. F. Moene, H. J. J. Jonker, G. J. F. van Heijst, H. J. H. Clercx, and B. J. H. van de Wiel, "Collapse of turbulence in stably stratified channel flow: A transient phenomenon," Q. J. R. Metereol. Soc. 141, 2137-2147 (2015).

${ }^{29}$ J. M. M. Donda, I. G. S. van Hooijdonk, A. F. Moene, G. J. F. van Heijst, H. J. H. Clercx, and B. J. H. van de Wiel, "The maximum sustainable heat flux in stably stratified channel flows," Q. J. R. Metereol. Soc. 142, 781-792 (2016).

${ }^{30}$ B. J. H. V. de Wiel, A. F. Moene, H. J. J. Jonker, P. Baas, S. Basu, J. M. M. Donda, J. Sun, and A. A. M. Holtslag, "The minimum wind speed for sustainable turbulence in the nocturnal boundary layer," J. Atmos. Sci. 69, 3116-3127 (2012).

${ }^{31}$ S. Komori, R. Nagaosa, Y. Murakami, S. Chiba, K. Ishii, and K. Kuwahara, "Direct numerical simulation of three-dimensional open-channel flow with zeroshear gas-liquid interface," Phys. Fluids A 5, 115-125 (1993).

${ }^{32}$ S. Komori, R. Kurose, K. Iwano, T. Ukai, and N. Suzuki, "Direct numerical simulation of wind-driven turbulence and scalar transfer at sheared gas-liquid interfaces," J. Turbul. 11, N32 (2010).

${ }^{33}$ J. Lee, J. Suh, H. J. Sung, and B. Pettersen, "Structures of turbulent openchannel flow in the presence of an air-water interface," J. Turbul. 13, N18 (2012).

${ }^{34}$ S. Kara, M. C. Kara, T. Stoesser, and T. W. Sturm, "Free-surface versus rigidlid LES computations for bridge-abutment flow,” J. Hydraul. Eng. 141, 04015019 (2015).

${ }^{35}$ B. J. H. V. de Wiel, E. Vignon, P. Baas, I. G. S. van Hooijdonk, S. J. A. van der Linden, J. A. van Hooft, F. C. Bosveld, S. R. de Roode, A. F. Moene, and C. Genthon, "Regime transitions in near-surface temperature inversions: A conceptual model," J. Atmos. Sci. 74, 1057-1073 (2017).
${ }^{36}$ P. Holmes, J. L. Lumley, G. Berkooz, and C. W. Rowley, Turbulence, Coherent Structures, Dynamical Systems and Symmetry, 2nd ed., Cambridge Monographs on Mechanics (Cambridge University Press, 2012).

${ }^{37}$ P. Davidson, Y. Kaneda, and K. Sreenivasan, Ten Chapters in Turbulence (Cambridge University Press, 2012).

${ }^{38}$ A. A. Townsend, "Equilibrium layers and wall turbulence," J. Fluid Mech. 11, 97-120 (1961).

${ }^{39}$ A. A. Townsend, The Structure of Turbulent Shear Flow (Cambridge University Press, 1976).

${ }^{40} \mathrm{~J}$. R. Taylor, "Numerical simulations of the stratified oceanic bottom boundary layer," Ph.D. thesis, University of California San Diego, 2008.

${ }^{41}$ C. Canuto, M. Y. Hussaini, A. Quarteroni, A. Thomas, Jr. et al., Spectral Methods in Fluid Dynamics (Springer Science \& Business Media, 1988).

${ }^{42} \mathrm{G}$. Blaisdell, E. Spyropoulos, and J. Qin, "The effect of the formulation of nonlinear terms on aliasing errors in spectral methods," Appl. Numer. Math. 21, 207-219 (1996).

${ }^{43}$ C. A. J. Fletcher, Computational Techniques for Fluid Dynamics 2: Specific Techniques for Different Flow Categories (Springer, 1991).

${ }^{44}$ A. W. Vreman and J. G. M. Kuerten, "Statistics of spatial derivatives of velocity and pressure in turbulent channel flow," Phys. Fluids 26, 085103 (2014).

${ }^{45}$ S. B. Pope, Turbulent Flows (Cambridge University Press, 2000).

${ }^{46} \mathrm{O}$. Flores and J. Jiménez, "Hierarchy of minimal flow units in the logarithmic layer," Phys. Fluids 22, 071704 (2010).

${ }^{47}$ A. Lozano-Durán and J. Jiménez, "Effect of the computational domain on direct simulations of turbulent channels up to $R e_{\tau}=4200$," Phys. Fluids 26, 011702 (2014).

${ }^{48}$ F. Lluesma-Rodríguez, S. Hoyas, and M. Perez-Quiles, "Influence of the computational domain on DNS of turbulent heat transfer up to $\operatorname{Re}_{\tau}=2000$ for $\operatorname{Pr}=0.71$," Int. J. Heat Mass Transfer 122, 983-992 (2018).

${ }^{49}$ J. Cuxart, C. Yagüe, G. Morales, E. Terradellas, J. Orbe, J. Calvo, A. Fernández, M. R. Soler, C. Infante, P. Buenestado, A. Espinalt, H. E. Joergensen, J. M. Rees, J. Vilá, J. M. Redondo, I. R. Cantalapiedra, and L. Conangla, "Stable atmospheric boundary-layer experiment in Spain (SABLES 98): A report," Boundary-Layer Meteorol. 96, 337-370 (2000).

${ }^{50} \mathrm{Z}$. Sorbjan, "Gradient-based scales and similarity laws in the stable boundary layer," Q. J. R. Metereol. Soc. 136, 1243-1254 (2010).

${ }^{51}$ O. Williams, T. Hohman, T. Van Buren, E. Bou-Zeid, and A. J. Smits, "The effect of stable thermal stratification on turbulent boundary layer statistics," J. Fluid Mech. 812, 1039-1075 (2017).

${ }^{52}$ J. Jiménez, "Turbulence and vortex dynamics," Notes for the Polytecnique Course on Turbulence, 2004.

${ }^{53} \mathrm{~N}$. Hutchins and I. Marusic, "Large-scale influences in near-wall turbulence," Philos. Trans. R. Soc., A 365(1852), 647-664 (2007).

${ }^{54} \mathrm{~N}$. Hutchins and I. Marusic, "Evidence of very long meandering features in the logarithmic region of turbulent boundary layers," J. Fluid Mech. 579, 1-28 (2007).

${ }^{55}$ J. C. R. Hunt, A. A. Wray, and P. Moin, "Eddies, streams, and convergence zones in turbulent flows," Studying Turbulence Using Numerical Simulation Databases, Technical Report CTR-S88, 1988, pp. 193-208.

${ }^{56} \mathrm{X}$. Dong, G. Dong, and C. Liu, "Study on vorticity structures in late flow transition," Phys. Fluids 30, 104108 (2018).

${ }^{57}$ G. Eitel-Amor, R. Örlü, P. Schlatter, and O. Flores, "Hairpin vortices in turbulent boundary layers," Phys. Fluids 27, 025108 (2015).

${ }^{58}$ N. E. Sujovolsky, P. D. Mininni, and A. Pouquet, "Generation of turbulence through frontogenesis in sheared stratified flows," Phys. Fluids 30, 086601 (2018). ${ }^{59}$ R. J. Adrian, "Hairpin vortex organization in wall turbulence," Phys. Fluids 19, 041301 (2007).

${ }^{60} \mathrm{~J}$. M. Hamilton, J. Kim, and F. Waleffe, "Regeneration mechanisms of near-wall turbulence structures," J. Fluid Mech. 287, 317-348 (1995).

${ }^{61} \mathrm{R}$. L. Coulter, "A case study of turbulence in the stable nocturnal boundary layer," Boundary-Layer Meteorol. 52, 75-91 (1990).

${ }^{62} \mathrm{Z}$. Sorbjan, "Local structure of turbulence in stably stratified boundary layers," J. Atmos. Sci. 63, 1526-1537 (2006).

${ }^{63}$ M. L. Waite and P. Bartello, "Stratified turbulence dominated by vortical motion," J. Fluid Mech. 517, 281-308 (2004). 
${ }^{64} \mathrm{M}$. L. Waite, "Direct numerical simulations of laboratory-scale stratified turbulence," in Modeling Atmospheric and Oceanic Flows: Insights from Laboratory Experiments and Numerical Simulations (Wiley, 2014), pp. 159-175.

${ }^{65}$ P. A. Davidson, Turbulence in Rotating, Stratified and Electrically Conducting Fluids (Cambridge University Press, 2013).

${ }^{66}$ T. M. Dillon and D. R. Caldwell, "The Batchelor spectrum and dissipation in the upper ocean," J. Geophys. Res.: Oceans 85, 1910-1916, https://doi.org/10.1029/jc085ic04p01910 (1980).
${ }^{67}$ S. H. Derbyshire, "Nieuwstadt's stable boundary layer revisited," Q. J. R. Metereol. Soc. 116, 127-158 (1990).

${ }^{68} \mathrm{~J}$. Jiménez and A. Pinelli, "The autonomous cycle of near-wall turbulence," J. Fluid Mech. 389, 335-359 (1999).

${ }^{69}$ S. Genieys and M. Massot, "From Navier-Stokes equations to OberbeckBoussinesq approximation: A unified approach," Paru comme un rapport interne de l'unité MAPLY (UMR 5585-Lyon) en 2001, 2001, http://maply.univlyonl.fr/publis/publiv/2001/331/publi.ps.gz. 